BULLETIN (New Series) OF THE

AMERICAN MATHEMATICAL SOCIETY

Volume 47, Number 1, January 2010, Pages 1-30

S 0273-0979(09)01274-9

Article electronically published on October 28, 2009

\title{
ON THE PROBLEM \\ OF RESOLUTION OF SINGULARITIES \\ IN POSITIVE CHARACTERISTIC \\ (Or: A proof we are still waiting for)
}

\author{
HERWIG HAUSER
}

\begin{abstract}
Assume that, in the near future, someone can prove resolution of singularities in arbitrary characteristic and dimension. Then one may want to know why the case of positive characteristic is so much harder than the classical characteristic zero case. Our intention here is to provide this piece of information for people who are not necessarily working in the field. A singularity of an algebraic variety in positive characteristic is called wild if the resolution invariant from characteristic zero, defined suitably without reference to hypersurfaces of maximal contact, increases under blowup when passing to the transformed singularity at a selected point of the exceptional divisor (a so called kangaroo point). This phenomenon represents one of the main obstructions for the still unsolved problem of resolution in positive characteristic. In the present article, we will try to understand it.
\end{abstract}

\section{INTRODUCTION}

The embedded resolution of singular algebraic varieties of dimension greater than 3 defined over fields of characteristic $p>0$ is still an open problem. The inductive argument which works in characteristic zero fails for positive characteristic. The main obstruction is the failure of maximal contact, which, in turn, manifests in the occurence of wild singularities and kangaroo points at certain stages of a sequence of blowups. At these points the standard characteristic zero resolution invariant increases instead of decreasing. The induction breaks down. No remedy has been found yet.

In this article, which is mostly expository, we will give a detailed discussion of the obstructions to resolution in positive characteristic. The description of wild singularities is based on the notion of oblique polynomials. These are homogeneous polynomials showing a specific behaviour under linear coordinate changes, which, in turn, determines them completely. Blowing up a wild singularity may cause the appearance of kangaroo points on the exceptional divisor. They represent one of the main problems for establishing the induction in positive characteristic.

Received by the editors November 17, 2008, and, in revised form, December 27, 2008, February 12, 2009, and July 10, 2009.

2010 Mathematics Subject Classification. Primary 14B05, 14E15, 12 D10.

Supported within the project P-18992 of the Austrian Science Fund FWF. The author thanks the members of the Clay Institute for Mathematics at Cambridge and the Research Institute for Mathematical Science at Kyoto for their kind hospitality.

(C)2009 American Mathematical Society 
The proofs and the technical details can be found in the original preprint Ha1, which is currently being revised and updated, cf. Ha2. While this work mainly addresses algebraic geometers with some experience in resolution matters, we will add footnote explanations and references for readers who are curious about recent developments but less familiar with the topic.

Sections A and B develop the overall outset of the resolution of singularities. Sections C and D then exhibit the specific problems related to fields of positive characteristic. These sections are written for a general audience. Starting with section $\mathrm{E}$, the reader will find more detailed information and precise statements.

A. Prelude for the nonexpert reader. Before getting into the actual material, let us tell you what resolution is about and why it is important (and, also, why it is so fascinating). Readers acquainted with the subject may proceed directly to the next but one section. A system of polynomial equations in $n$ variables has a zeroset - the associated algebraic variety $X$ - whose structure can be quite complicated and mysterious. You may think of the real or complex solutions of an equation like

$$
441\left(x^{2} y^{2}+y^{2} z^{2}+x^{2} z^{2}\right)=\left(1-x^{2}-y^{2}-z^{2}\right)^{3}
$$

(see Figure 2 at the end of the article for a visualization of this surface). The geometry of varieties shows all kinds of local and global patterns which are difficult to guess from the equation. In particular, there will be singularities. These are the points where $X$ fails to be smooth (i.e., where $X$ is not a manifold). At those points the Implicit Function Theorem (IFT) cannot be used to compute the nearby solutions. As a consequence, it is hard (also for computers) to describe correctly the local shape of the variety at its singular points.

Resolution of singularities is a method for understanding where singularities come from, what they look like, and what their internal structure is. The idea is quite simple: when you take a submanifold $X$ of a high-dimensional ambient space $M$ and then consider the image $X^{\prime}$ of $X$ under the projection of the ambient space onto a smaller space $M^{\prime}$, you most often create singularities on $X^{\prime}$. The Klein bottle is smooth as a submanifold of $\mathbb{R}^{4}$, but there is no smooth realization of it in $\mathbb{R}^{3}$. You necessarily have to accept self-intersections. Similarly, if you project a smooth space curve onto a plane in the direction of a tangent line at one of its points, the image curve will have singularities.

Which singular varieties can we obtain by such "projections"? The answer is simple: All!

Theorem (Hironaka 1964). Every algebraic variety over $\mathbb{C}$ is the image of a manifold under a suitable projection. Such a manifold and map can be explicitly constructed (at least theoretically).

For a geometer, this is quite amazing. For an algebraist, this is even more striking, since it means that it is possible to solve polynomial equations up to the Implicit Function Theorem. The applications of this result are numerous (it would be worth listing all the theorems whose proofs rely on resolution). The reason is that, for smooth varieties, a lot of machinery is available to construct invariants and associated objects (zeta-functions, cohomology groups, characteristic classes, extensions of functions and differential forms, ...). As the projection map consists of a sequence of relatively simple maps (so-called blowups), there is a good chance 
of carrying these computations over to singular varieties. Which, in turn, is very helpful in understanding them better.

Resolution is well established over fields of characteristic zero (with nowadays quite accessible proofs) but still unknown in positive characteristic (except for dimensions up to 3). Why bother about this? First, because (almost) everybody expects resolution to be true also in characteristic $p$. As the characteristic zero case was already a great piece of work (built on a truly beautiful concatenation of arguments), it is an intriguing challenge for the algebraic geometry community to find a proof that does not use the assumption of characteristic zero. But there is more to it: many virtual results in number theory and arithmetic are just waiting to become true by having at hand resolution in positive characteristic 1 Again, it would be interesting to produce a list.

Another important feature of such a proof is our understanding of solving equations in characteristic $p$. If we agree not to aim at one-stroke solutions but to simplify the equation step by step (for instance, using blowups) until we can see the solution (again, modulo IFT), there appears this delicate matter of understanding local coordinate changes in the presence of the Frobenius homomorphism. Phrased in very down-to-earth terms this means, How do you measure whether a polynomial is, up to coordinate changes and up to adding pth power polynomials, close or far from being a monomial? This is less naive than it may sound: it is an extremely tough question (it has resisted solution for over 50 years), and it lies at the very heart of the resolution of singularities in characteristic $p$. A meaningful proposal for such a measure (which should be compatible with blowups in a well-defined sense) could break open the wall behind which we suspect there will be a proof of resolution in positive characteristic. The rest would be mainly technicalities.

In the present article we will see some of these "elementary" characteristic $p$ features, and we will make them very explicit. Of course it would be nice to have in parallel the conceptual counterparts of these constructions and phenomena, but this would require much more space and effort (for both the reader and the writer). As a consolation, the problems will be so concrete that everybody with a minimum talent in algebra will be tempted to attack them. The more geometrically oriented reader is referred to the survey $[\mathrm{FH}]$ for various visualizations of the resolution of surface singularities.

At the end of this paper, we briefly describe the present state of research in resolution of singularities in positive characteristic and arbitrary dimension (work of Hironaka, Villamayor, Kawanoue and Matsuki, Włodarczyk).

\section{Overall discussion}

B. Resumé of techniques and results. This section will explain the main resolution devices that work independently of the characteristic. The material has become classical, with many excellent references. After this survey section we will return to the failure of maximal contact and the description of wild singularities and kangaroo points in positive characteristic.

By far the most important modification of a variety is given by the concept of blowup. Every blowup comes with a center (a carefully chosen subvariety of our variety) which is the locus of points where the variety is actually modified. Outside

\footnotetext{
${ }^{1}$ De Jong's theory of alterations, valid in arbitrary characteristic but slighty weaker than resolution, already produced a swarm of such results, cf. the next section.
} 
the center, the variety remains untouched. The center itself is replaced by a larger subvariety, which affects the way in which the variety approaches this locus. The hope is that blowups gradually improve the singularities of the varieties until, after possibly many steps, all singularities are eliminated. Whereas this elimination is granted in characteristic zero if one chooses the correct sequence of blowups, the situation is much more delicate in positive characteristic. The main difficulty is to measure the complexity of a singularity by an invariant (usually a lexicographically ordered vector of integers) in a way such that after each blowup (chosen suitably) the invariant has decreased. This is precisely the theme of this article: the invariant that works well in zero characteristic tends to behave erratically in positive characteristic.

Apart from blowups, the two other main characteristic-free techniques are normalizations and alterations. They will be described at the end of this section.

Blowups. We will start with a short introduction to blowups. There are many equivalent ways to define them (see, e.g., [EiH, Ha5]). We shall choose the most geometric and intuitive description. By an affine variety we shall always understand a subset of affine space $\mathbb{A}_{K}^{n}$ over a field $K$ to be the zeroset $X$ of a bunch of polynomials in $n$ variables. We do not assume that $X$ is irreducible. The coordinate ring $K[X]$ is the quotient of $K\left[x_{1}, \ldots, x_{n}\right]$ by the ideal $I_{X}$ generated by these polynomials 2

A blowup of $X$ is a new variety $X^{\prime}$, the blowup or transform of $X$ together with a morphism $\pi: X^{\prime} \rightarrow X$, the blowup map. Any blowup is determined by its center $Z$. This is a closed, nonempty subvariety $Z$ of $X$, usually smooth and included in the singular locus $\operatorname{Sing}(X)$ of $X$ (the locus of points where $X$ is not smooth; below we define the blowup with respect to an arbitrary ideal). The definition of $X^{\prime}$ and $\pi$ does not need the embedding $X \subset \mathbb{A}^{n}$, but the explanation is easier if we use it. Moreover, we shall assume that we are given a projection $\rho: X \rightarrow Z$ (again, this is not substantial, but makes things simpler). We should think of $X$ as being fibered by transversal sections along $Z$. If $Z$ is just a point, $\rho$ is the constant map. If $Z$ is a line, then $\rho$ is typically the restriction to $X$ of a linear orthogonal projection from $\mathbb{A}^{n}$ to $Z$ (having chosen some scalar product on $\mathbb{A}^{n}$ ). For any point $a$ of $X$ not in $Z$ there is a unique line in $\mathbb{A}^{n}$, call it $\ell_{a}$, passing through $a$ and its projection point $\rho(a)$ in $Z$. This is just the secant line through $a$ and $\rho(a)$, and it belongs to the space $L\left(\mathbb{A}^{n}\right)$ of all lines in $\mathbb{A}^{n}$. In particular, the notion of limit line makes sense when $a$ approaches $\rho(a)$ inside the fiber of $\rho$.

The idea of blowups consists in pulling apart $X$ inside a larger ambient space. As $\ell$ is defined on $X \backslash Z$ and takes values in $L\left(\mathbb{A}^{n}\right)$, the graph $\Gamma(\ell)$ of $\ell$ will be an algebraic subset of $(X \backslash Z) \times L\left(\mathbb{A}^{n}\right)$. It allows us to see $X \backslash Z$ embedded into $(X \backslash Z) \times L\left(\mathbb{A}^{n}\right)$ via $a \rightarrow\left(a, \ell_{a}\right)$. We now extend this embedding to the whole of $X$. The result will no longer be an embedding, but rather a subvariety $X^{\prime}$ of $X \times L\left(\mathbb{A}^{n}\right)$ which projects onto $X$. Above points $a$ of $Z$, there will be, in general, several points in $X^{\prime}$, namely all the limiting positions of secants through $a$. Taking the limit of the secants $\ell_{a}$ as $a \in X \backslash Z$ approaches $Z$ corresponds to adding the boundary points of $\Gamma(\ell) \subset(X \backslash Z) \times L\left(\mathbb{A}^{n}\right)$ when considered as a subset of $X \times L\left(\mathbb{A}^{n}\right)$. More precisely, let $X^{\prime}$ be the Zariski closure of $\Gamma(\ell)$ in $X \times L\left(\mathbb{A}^{n}\right)$, i.e., the smallest algebraic subset

\footnotetext{
${ }^{2}$ All of what follows can be defined for arbitary schemes, the center being a closed subscheme. See the paragraphs after the examples below for a more conceptual definition of blowups.
} 
containing $\Gamma(\ell)$. Then $X^{\prime}$ will be the blowup of $X$ along $Z$, and $\pi: X^{\prime} \rightarrow X$ is the restriction of the projection $X \times L\left(\mathbb{A}^{n}\right) \rightarrow X$.

Intuitively, we can interpret $\ell(a)$ as the "height" of the point on $X^{\prime}$ projecting to $a \in X \backslash Z$. Above points $a$ in $Z$, this height will in general be multivalued. All this can be made very precise and has both algebraic and axiomatic interpretations (see the definition after the next examples and the references). For the moment, we apply this technique to specific geometric situations. As the blowup map will be an isomorphism over $X \backslash Z$ (by definition), and since we have no need to modify the smooth points of $X$, we shall always choose the center $Z$, as mentioned earlier, inside the singular locus $\operatorname{Sing}(X)$ of $X$.

Example 1. Let $X$ be the cone in the three-dimensional real affine space $\mathbb{A}_{\mathbb{R}}^{3}$ of equation $x^{2}+y^{2}=z^{2}$, and let the center $Z$ be its unique singular point, the origin 0 . We claim that the blowup $X^{\prime}$ of $X$ in 0 is the cylinder $x^{2}+y^{2}=1$. This can be checked algebraically, but it is nicer to convince ourselves by a geometric argument. See the lines in $\mathbb{A}^{3}$ through 0 as elements of projective space $\mathbb{P}^{2}$. Our height function $\ell: X \backslash 0 \rightarrow \mathbb{P}^{2}$ is defined by associating to any point $a \in X \backslash 0$ the line through $a$ and 0 (which is just a generating line of the cone). As $a$ moves on the cone straight toward 0 , the line $\ell_{a}$ will always be the same, so the map $\ell$ is constant on the lines of $X$. Clearly, the limiting positions form a circle, and we conclude that $X^{\prime}$ is indeed the cylinder.

Example $1^{\prime}$. Take for $X$ the plane curve of equation $x^{2}=y^{2}+y^{3}$, the node. The natural center to choose is the singular point 0 . The same reasoning as before shows that $X^{\prime}$ is a smooth curve. Also, taking the Cartesian product $Y$ of $X$ with a perpendicular axis in $\mathbb{A}^{3}$ (seeing $\mathbb{A}^{2}$ as the plane $\mathbb{A}^{2} \times 0$ in $\mathbb{A}^{3}$ ) will pose no problems: the center $Z$ is now the $z$-axis, we fiber $\mathbb{A}^{3}$ by the planes $\mathbb{A}^{2} \times\{t\}$ with $t$ varying in $\mathbb{A}^{1}$, and get a blowup $Y^{\prime}$ of $Y$ which is the Cartesian product of the blowup $X^{\prime}$ of $X$ with the $z$-axis.

Example 2. The cusp $X$ of equation $x^{2}=y^{3}$ is slightly more complicated to treat. The blowup of $X$ with center the origin 0 will be a space curve $X^{\prime}$ in the threedimensional ambient space $\mathbb{A}^{2} \times \mathbb{P}^{1}$. As $X$ has just one limit of secants at 0 (the $y$-axis), there is precisely one point on $X^{\prime}$ sitting above $0 \in X$. Call it $a^{\prime}$. We have to check whether $X^{\prime}$ is smooth or singular at $a^{\prime}$. Unfortunately, this can no longer be done by purely geometric methods, and we have to resort to algebra. Points $a$ on $X$ are of the form $\left(t^{3}, t^{2}\right)$, the respective secant, taken as an element in $\mathbb{P}^{1}$, has projective coordinates $\left(t^{3}: t^{2}\right)=(t: 1)$, so that the points of $X^{\prime}$ are parametrized by $t \rightarrow\left(t^{3}, t^{2}, t\right)$. Hence $X^{\prime}$ is smooth at $a^{\prime}=0$. The same computation applied to the "sharper" cusp $Y$ defined by $x^{2}=y^{5}$ yields for $Y^{\prime}$ the parametrization $\left(t^{5}, t^{2}, t^{3}\right)$. This shows that $Y^{\prime}$ is still a singular curve. Project $Y^{\prime}$ to the plane $\mathbb{A}^{2}$ by forgetting the first component. The image curve is the ordinary cusp $X$. By construction, $X$ is isomorphic to $Y^{\prime}$. Therefore, another point blowup suffices to resolve $Y^{\prime}$.

There is an algebraic and slightly more general notion of blowup which is related to an arbitrary ideal $N$ in $K\left[x_{1}, \ldots, x_{n}\right]$ (now $K$ can be any field). The geometric version above is recovered by taking for $N$ the radical ideal $I_{Z}$ defining $Z$ in $\mathbb{A}^{n}$. Let $g_{1}, \ldots, g_{k}$ be a system of generators of $N$, and let $Z \subset \mathbb{A}^{n}$ be the common zeroset of the $g_{i}$ (which coincides with the subvariety of $\mathbb{A}^{n}$ defined by $N$ ). Then 
the map

$$
g: \mathbb{A}^{n} \backslash Z \rightarrow \mathbb{P}^{k-1}, \quad a \rightarrow\left(g_{1}(a): \cdots: g_{k}(a)\right)
$$

is well defined. The Zariski closure $\widetilde{\mathbb{A}}^{n}$ of its graph in $\mathbb{A}^{n} \times \mathbb{P}^{k-1}$ is defined as the blowup of $\mathbb{A}^{n}$ with center $N$. It is easy to see that $\widetilde{\mathbb{A}}^{n}$ is a variety of dimension $n$, and isomorphic to the blowup defined geometrically above in case $N$ is the radical ideal of the subvariety $Z$ of $\mathbb{A}^{n}$. In particular, $\widetilde{\mathbb{A}}^{n}$ is smooth if $Z$ is smooth. The restriction to $\widetilde{\mathbb{A}}^{n}$ of the first projection $\mathbb{A}^{n} \times \mathbb{P}^{k-1} \rightarrow \mathbb{A}^{n}$ yields the blowup map $\pi: \widetilde{\mathbb{A}}^{n} \rightarrow \mathbb{A}^{n}$.

Embedded resolution. We will have to distinguish between embedded and nonembedded resolution. To explain the difference, let our singular variety be irreducible and embedded in some smooth ambient space $W$, say, for simplicity, $W=\mathbb{A}^{n}$. Let $Z$ be a subvariety of $X$ (our chosen center of blowup). It is a general fact that the blowup $X^{\prime}$ of $X$ along $Z$ can be constructed from the blowup of $W$ along $Z$. To this end, denote by $\pi: W^{\prime} \rightarrow W$ the blowup map, and consider the inverse image $X^{*}=\pi^{-1}(X)$ of $X$ under $\pi$. The variety $X^{*}$ is called the total transform of $X$. It turns out that $X^{*}$ has two components. The first is the exceptional divisor $E=\pi^{-1}(Z) \subset W^{\prime}$ given by the pull-back of the center. It is a hypersurface in $W^{\prime}$ which contracts under $\pi$ to $Z$, whereas outside $E$ the map $\pi$ is an isomorphism onto $X \backslash Z$. The second component, say $X^{\prime}$, is the geometrically interesting object. It coincides with the blowup of $X$ along $Z$ and is called the strict transform of $X$ under $\pi$. Taking the inverse image $\pi^{-1}(X \backslash Z)$ of $X \backslash Z$ in $W^{\prime}$, the Zariski closure of $\pi^{-1}(X \backslash Z)$ in $W^{\prime}$ gives $X^{\prime}$.

An embedded resolution of $X \subset W$ is a birational proper morphism $\pi: \widetilde{W} \rightarrow W$ so that the total transform $X^{*}$ is a variety with at most normal crossings 3 This signifies that the strict transform $X^{\prime}$ of $X$ under $\pi$ is smooth and transversal to the components of the exceptional divisor $E$ in $\widetilde{W}$.

In contrast, a nonembedded resolution of $X$ is just a birational proper morphism $\varepsilon: \widetilde{X} \rightarrow X$ with $\widetilde{X}$ smooth. It does not take into account the embedding of $X$ but considers $X$ as an abstract variety. We should think of $\varepsilon$ as a parametrization of the singular variety $X$ by the smooth variety $\widetilde{X}$. A basic result in birational geometry says that any projective birational morphism is given as a single blowup of $X$ along a center $Z$ defined by a possibly very complicated ideal. In particular, this holds for any resolution, where now the center should be supported on $\operatorname{Sing}(X)$. Even in the first nontrivial examples it is not clear how to define such a center $a b$ initio in order to get the required resolution via the induced blowup.

For many applications one needs embedded resolution. The concept has a variant known as log-resolution of varieties, respectively principalization or monomialization of ideals - the ideal will be the one defining $X$ in $W 4$ If a nonembedded resolution $\varepsilon: \widetilde{X} \rightarrow X$ is given by a sequence of blowups in certain centers and if we have an embedding $X \subset W$ of $X$ into a smooth variety $W$, one may take the successive blowups of $W$ defined by these centers. This yields a birational morphism

\footnotetext{
${ }^{3}$ Birational morphism: A map given locally by quotients of polynomials inducing an isomorphism onto a dense open subset of the target space. Proper: The preimage of compact sets is compact. Normal crossings: Locally, the variety is, up to isomorphism, a union of coordinate subspaces, or, equivalently, can be defined by a monomial ideal.

${ }^{4}$ For a log-resolution one requires that the total transform is in addition a divisor, say, a hypersurface of the smooth ambient space. This can be achieved from an embedded resolution by an extra blowup with center the entire strict transform of the variety.
} 
$\pi: \widetilde{W} \rightarrow W$ of smooth varieties together with an embedding $\widetilde{X} \subset \widetilde{W}$. At this stage, $\widetilde{X}$ need not meet the exceptional divisor $E$ of $\pi$ transversally (here, $E$ is defined as the subvariety of $\widetilde{W}$ where $\pi$ is not an isomorphism). But then one can apply further blowups to $\widetilde{W}$ until all components of the transform of $\widetilde{X}$ and $E$ do meet transversally, which then provides an embedded resolution of $X$ in $W$.

Small dimensions. Let us now turn to resolution of curves, surfaces, and three-folds in arbitrary characteristic.

The resolution of curves is governed by the fact that all singular points are isolated, so that only point blowups have to be considered. One can choose any of the singular points as center. The order in which these are taken does not matter. So the only problem is to show that after finitely many such blowups the resulting curve is smooth (and, for the embedded resolution, transversal to the exceptional divisor). This is done by defining a local invariant at each of the singular points of the curve and showing that, after one blowup, this invariant has dropped at all singular points sitting above the center. Various invariants for this task have been proposed and work; see the first chapter of Kollár's book describing thirteen ways to resolve curve singularities $[\mathrm{Ko}$. The most frequent invariant for plane curves consists of two numbers $(o, s)$ (considered lexicographically), where $o$ is the order of the Taylor expansion of the defining equation at the point and $s$ is the first characteristic number (which we do not define here). For a detailed discussion of this invariant in arbitrary characteristic and the proof that it drops under blowup, you may consult the survey $[\mathrm{HR}$.

Let us next consider singular surfaces that are embedded as hypersurfaces in a smooth three-dimensional ambient space. The singular locus consists now of isolated points and (possibly) curves, which themselves can be singular 5 The isolated points can be taken as the center of a blowup as before, with the task to exhibit numerically the improvement of the singularities after the blowup. The first complication is due to the fact that an isolated singularity may produce a whole curve of singular points under blowup on the transformed surface. The second complication stems from the curves along which the surface is singular. If the curve is smooth, it can be taken as center, with the hope of getting an improvement. If it is singular, its singular points are the only reasonable centers, because blowups whose center is a singular curve are very difficult to control. So we start with point blowups. By resolution of curves, finitely many blowups resolve these curves (i.e., make them smooth). On the way, new curves may appear in the singular locus of the surface. Zariski has shown that they are always smooth. This allows us to conclude that after finitely many blowups the singular locus of the surface consists of isolated points and smooth curves that, moreover, intersect transversally. From that point on we also take curves as centers: any component of the singular locus of the surface may be chosen (again, the order does not matter, as Zariski showed).

It remains for us to show that the sequence of blowups (which is geometrically motivated) does indeed resolve all singularities. To this end it suffices to show that the order of the defining equation must drop after finitely many blowups. This problem and the solution to it are known as the theorem of Beppo Levi. Again, an

\footnotetext{
${ }^{5}$ In practice, one considers instead of the singular locus the usually smaller top locus defined earlier as the set of points where the local order of the defining equation is maximal; the same reasoning applies with slight modifications.
} 
invariant that drops after each blowup has to be defined. There are several proposals. Zariski was able to construct one for characteristic zero, and Abhyankar was the first to give a proof of termination in positive characteristic [Za, Ab1]. Hironaka later defined a different and characteristic-free invariant based on the Newton polyhedron of the defining equation [Hi4, Ha3]. The construction is quite special and does not seem to apply to higher dimensions. Hauser and Wagner showed, relying on a proposal of Zeillinger, that the nowadays standard characteristic zero invariant of Villamayor, Bierstone and Milman, and successors that works in arbitrary dimension (but may increase for blowups in positive characteristic) can be modified suitably in the case of surfaces by subtracting a bonus from it. This bonus is a small correction term which takes values between 0 and $1+\delta$ according to the internal structure of the defining equation; see the section on the resolution of surfaces. The modified invariant then decreases after each blowup and thus provides an induction argument [HW]. Quite recently, Cossart, Jannsen and Saito established resolution for surfaces that are not necessarily hypersurfaces over a field by extending Hironaka's construction to arbitrary excellent two-dimensional schemes CJS. It turns out that all these techniques actually allow us to produce an embedded resolution (since we are working with the defining equations of the surface). In contrast, Lipman's proof of resolution of surfaces via normalization plus blowups yields a nonembedded resolution $\mathrm{Lp} 16$

The situation for three-folds is much more involved. At the moment only nonembedded resolution is established (in arbitrary characteristic). The proof relies vitally on the embedded resolution of surfaces. Abhyankar gave a long proof (more than 500 pages) that is scattered over several papers and requires that the characteristic of the algebraically closed ground field is greater than 5. Cutkosky was then able to make this proof much more transparent and to reduce it to less than forty pages $\mathrm{Cu}$. In Cutkosky's paper, Abhyankar's work is described in great detail, giving all necessary references. Cossart and Piltant succeeded in removing the restriction on the characteristic and the algebraic closedness of the ground field. The resulting proof is rather long and challenging $\mathrm{CP} 1, \mathrm{CP} 2$, based on ideas of $\mathrm{Co}$.

Normalization. All the above approaches use in some way or other the modification of a variety by blowups. Let us now describe two alternatives.

An important way to improve the singularities of a variety is by means of its normalization. This is an extremely elegant, characteristic independent method to get rid of all components of the singular locus of codimension one in the variety (e.g., curves in the singular locus of a surface). One says that the variety becomes regular in codimension one. The construction does not look at the embedding.

The normalization is defined through the integral closure of rings. Assume that $X$ is an irreducible algebraic subset of affine space $\mathbb{A}^{n}$ over the ground field $K$, and let $R$ be the coordinate ring of $X$ whose elements are the polynomial functions on $X$. The ring $R$ is a finitely generated $K$-algebra and an integral domain. Let $Q$ be its field of fractions (the function field of $X$ ). Now recall that any morphism $f: X^{\prime} \rightarrow X$ of varieties induces a dual ring homomorphism $f^{*}: R \rightarrow R^{\prime}$ between the coordinate rings given by composition with $f$. If the morphism $f$ is birational, the map $f^{*}$ is injective and induces an isomorphism of function fields $Q \cong Q^{\prime}$.

\footnotetext{
${ }^{6}$ The proof selects among all normal varieties proper over the ground field and birational to $X$ one of minimal arithmetic genus, shows that all its singularities are pseudo-rational, and then resolves these by point blowups.
} 
Identifying $Q^{\prime}$ with $Q$, the morphism $f$ can then be read as a ring extension $R \subset$ $R^{\prime} \subset Q$. This observation suggests that we look at overrings of $R$ inside $Q$ that are again finitely generated $K$-algebras (in order to be the coordinate ring of a variety) and so that the corresponding variety is "closer" to a smooth variety than $X$.

One answer to this approach is the integral closure $\bar{R}$ of $R$ in $Q$. It can be shown that $\bar{R}$ is a finitely generated $K$-algebra and that the extension $R \subset \bar{R}$ is finite. Therefore, $\bar{R}$ is the coordinate ring of a variety $\bar{X}$, and the inclusion $R \subset \bar{R}$ defines a finite morphism $\bar{X} \rightarrow X$, the normalization map. The variety $\bar{X}$ is normal (its coordinate ring is integrally closed), in particular, it is regular in codimension one. For curves, this signifies smoothness (giving a nonembedded resolution); for surfaces we will only have isolated singularities (which are good for many purposes, but not yet a resolution). It can be shown that iterated compositions of normalizations and point blowups allow us to resolve surfaces.

Alterations. The last method that we shall mention in this Introduction is the notion of alterations introduced by de Jong dJ. It works in all characteristics but yields a resolution only up to a finite map. This, however, is sufficient for many applications $\mathrm{Be}$.

Let us briefly describe the idea. Whereas a modification of a variety $X$ is a birational proper morphism $\pi: X^{\prime} \rightarrow X$ yielding an isomorphism of function fields, an alteration is a proper, surjective morphism that induces a finite extension of function fields. Geometrically speaking, $\pi$ is an isomorphism, respectively a finite morphism over a (dense) open subset $U$ of $X$ (generic isomorphism, respectively generically finite morphism). A modification is a birational alteration, and an alteration factors into a modification followed by a finite map.

De Jong shows that any variety (say, over an algebraically closed field) admits an alteration $\varepsilon: X^{\prime} \rightarrow X$ with $X^{\prime}$ smooth (and quasi-projective) $\mathrm{dJ}, \mathrm{Be}, \mathrm{AO}$. For the proof by induction one needs a stronger and more precise statement: If $S$ is a closed subvariety of $X$, the alteration $\varepsilon$ can be chosen together with an open immersion $i: X^{\prime} \subset Y$ into a projective and smooth $Y$ so that the union $i\left(\varepsilon^{-1}(S)\right) \cup\left(Y \backslash X^{\prime}\right)$ forms a normal crossings divisor in $Y$.

The method of proof is opposite to the resolution proofs via blowups: After a preliminary alteration which allows us to assume $X$ to be projective and normal, the variety $X$ is fibered in curves by constructing a suitable morphism to a variety $P$ of dimension one less than the dimension of $X$. This may create singularities in the fibres which lie outside the singular locus of $X$. Then, using the theory of semistable reduction, a further alteration together with induction on the dimension of the base space reduces to the case where the fibres have at most nodal singularities (i.e., are defined locally by $x y=0$ ), and the singular fibres sit only over the points of a normal crossings divisor of $P$. The situation has then become so explicit that it can be treated by toric methods, finally yielding an alteration $\varepsilon: X^{\prime} \rightarrow X$ of $X$ with the required properties.

This concludes our summary on resolution. We now turn to the main theme of the article, the obstructions to the resolution of singularities in positive characteristic and arbitrary dimension.

C. Failure of maximal contact. There is a concrete reason why resolution is more difficult in positive characteristic: The behaviour of the singularities under blowup is much more erratic than in characteristic zero. Therefore it is harder to 
pinpoint and then measure a continuous improvement of the singularities yielding eventually to a resolution. In this section we explain this phenomenon. Some preliminary material is necessary.

For the ease of the exposition, we restrict our discussion to hypersurfaces $X$ defined by one equation $f=0$ in a smooth ambient space $W$ (e.g., affine space $\left.\mathbb{A}^{n}\right)$. Fix a point $a$ of $X$. Then $X$ is smooth at $a$ if and only if the order of the Taylor expansion of $f$ at $a$ is 1, i.e., if the expansion starts with a linear term. If $a$ is a singular point of $X$, the order is at least 2. Denote by $\operatorname{Sing}(X)$ the set of all singular points of $X$. This is an algebraic subset, called the singular locus of $X$; it is defined by the vanishing of the partial derivatives of $f$. The complexity of the singularity of $X$ at a point $a \in \operatorname{Sing}(X)$ is related to the order of vanishing of $f$ at $a$. Denote this number by $\operatorname{ord}_{a} X$, and by $\operatorname{ord}(X)$ the maximal value of $\operatorname{ord}_{a} X$ on $X$. As $\operatorname{ord}_{a} X$ is an upper semicontinuous function in $a$ and $X$ is a Noetherian space with respect to the Zariski topology, $\operatorname{ord}(X)$ is finite and the set $\operatorname{top}(X)$ of points of $X$ where $\operatorname{ord}_{a} X$ attains its maximum $\operatorname{ord}(X)$ is an algebraic subset. This top locus collects the "worst" singularities of $X$. Zariski calls it the equimultiple locus [Za]. The objective of the resolution process is to make ord $(X)$ drop by a sequence of blowups in well-chosen centers until it becomes 1 . Then $X$ will have become everywhere smooth 7

As blowups are isomorphisms outside the center, they will not change the local order of $X$ there. Since we are only concerned in a first instance to improve $X$ along $\operatorname{top}(X)$, the natural choice of center is therefore $Z=\operatorname{top}(X)$. The problem is that in general the top locus may itself be singular. Blowing up the smooth ambient space $W$ in a singular center creates a new ambient space $W^{\prime}$ which now may be singular and whose singularities can be hard to control. It is then unknown how to measure a possible improvement of the transform $X^{\prime}$ of $X$ in $W$. Therefore we are confined to choose always smooth centers $Z$. Something nice happens.

Fact. Let $Z \subset \operatorname{top}(X)$ be a smooth center, let $\pi: W^{\prime} \rightarrow W$ be the induced blowup with (strict) transform $X^{\prime}$ of $X$ in $W^{\prime}$. Let $a$ be a point in $Z$, and let $a^{\prime}$ be a point in $E=\pi^{-1}(Z) \subset W^{\prime}$ mapping under $\pi$ to $a$. Then

$$
\operatorname{ord}_{a^{\prime}} X^{\prime} \leq \operatorname{ord}_{a} X \text {. }
$$

In particular, we get $\operatorname{ord}\left(X^{\prime}\right) \leq \operatorname{ord}(X)$ for the maximum value of the local orders. This says that the complexity of the singularities of $X$ does at least not get worse. If $\operatorname{ord}\left(X^{\prime}\right)<\operatorname{ord}(X)$, we can apply induction. If $\operatorname{ord}\left(X^{\prime}\right)=\operatorname{ord}(X)$, there will be at least one point $a^{\prime} \in E$ with image $a \in Z$ and such that $\operatorname{ord}_{a^{\prime}} X^{\prime}=\operatorname{ord}_{a} X$. We call such points equiconstant points (classically, they are also called infinitely near points). They are the points where induction cannot be directly applied. Some refined argument is necessary.

One might hope that $\operatorname{ord}(X)$ always drops. This is immediately seen to be too optimistic, equality may indeed occur. One situation where equality must occur is the case when $\operatorname{top}(X)$ is singular. As the center $Z$ is required to be smooth, $Z$ is then strictly included in $\operatorname{top}(X)$. Therefore, $\operatorname{ord}_{a^{\prime}} X^{\prime}$ remains constant equal to $\operatorname{ord}_{a} X$ for all points $a^{\prime}$ above $a \in \operatorname{top}(X) \backslash Z$. Hence ord $\left(X^{\prime}\right)=\operatorname{ord}(X)$. Moreover, by the above fact and the upper semicontinuity of the order, it follows

\footnotetext{
${ }^{7}$ For an embedded resolution, one has to consider the total transform of $X$ and try to make it into a normal crossings variety. It is not known how to measure efficiently the "distance" of a singularity from being normal crossings.
} 
that $\operatorname{ord}_{a^{\prime}} X^{\prime}=\operatorname{ord}_{a} X$ also holds for all points $a^{\prime}$ above $a \in Z$. So there is no obvious improvement.

To respond to this quandary, one may try to make first $Y=\operatorname{top}(X)$ smooth by some auxiliary blowups, in order to take it afterward as the center of the next blowup. This fails in two directions: First, the blowup $Y^{\prime}$ of $Y$ need not coincide with the top locus of the transform $X^{\prime}$ of $X$. New and even singular components may pop up; see [Ha7] for an explicit example. So resolving $Y$ (for instance by induction on the dimension) does not really help to make top $(X)$ smooth (except for surfaces). Secondly, even if $\operatorname{top}(X)$ were smooth and would be taken as center, it can be shown that ord $(X)$ may not drop under the respective blowup.

From this analysis we learn that the main problem sits in the appearance of equiconstant points on the transform $X^{\prime}$ after a blowup. There, the order of $X$ has not dropped, and some refined measure for the improvement of the singularities has to be designed (provided that an improvement-as we hope-has occurred; this also depends on the correct choice of the center, a question which we will not address here).

The next step is to study in more detail the equiconstant points, especially their location on $X^{\prime}$. This may help us to understand better how the singularities transform under blowup when the order remains the same. So fix a point $a \in Z$ in the center $Z \subset \operatorname{top}(X)$ of a given blowup $\pi: W^{\prime} \rightarrow W$, and let $a^{\prime} \in E$ be an equiconstant point of $X^{\prime}$ above $a$. Zariski already observed that there exists, locally in a neighborhood of $a$ in $W$, a smooth hypersurface $V$ containing $a$ whose transform $V^{\prime}$ under $\pi$ contains all equiconstant points $a^{\prime}$ above $a[\mathrm{Za}$. This restricts considerably the location of these points. Zariski describes quite explicitly all such hypersurfaces.

Now comes the distinction between zero and positive characteristic. In zero characteristic Abhyankar and Hironaka observed 8 that $V$ can be chosen even so that its transform $V^{\prime}$ not only contains all equiconstant points but moreover has itself a transform $V^{\prime \prime}$ containing all equiconstant points $a^{\prime \prime}$ sitting above $a^{\prime}$. And this continues like this until the order of $X$ drops. It is thus possible to capture the whole sequence of equiconstant points above $a$ by one hypersurface together with its transforms. Such local smooth hypersurfaces, which accompany the resolution process, are called hypersurfaces of maximal contact (and are known as Tschirnhausen transformations in the terminology of Abhyankar). They play the crucial role for the resolution of singularities in characteristic zero and are defined locally at each point of interest. One may construct on them a new, subordinate resolution problem, call it $X_{-}$in $V$. Formulating this descent properly is not easy but can be done. The resolution of $X_{-}$in $V$ exists by induction on the dimension (this induction tells us also how to choose the centers). Having resolved $X_{-}$it can be proven that the singularities of $X$ in the original ambient space $W$ must also have improved (in a precisely defined way). This is the key argument in characteristic zero.

In positive characteristic, this argument fails drastically: hypersurfaces of maximal contact need not exist. There are examples of (e.g., two-dimensional) hypersurfaces with isolated singularities together with a sequence of (point) blowups where any local smooth hypersurface $V$ passing through the singularity eventually loses

\footnotetext{
${ }^{8}$ According to rumors, one breakthrough happened at the end of the 1950 s on the occasion of a four day visit of Hironaka at Abhyankar's house.
} 
the sequence of equiconstant points sitting above the initial point $\mathrm{Na1}$, Ha2]. This prohibits the application of the same descent in dimension as in characteristic zero.

Still, for a single blowup, one can choose, by Zariski's observation, locally at $a \in \operatorname{top}(X)$ a smooth hypersurface $V$ in $W$ whose transform $V^{\prime}$ contains the equiconstant points $a^{\prime}$ of $X^{\prime}$ in $W^{\prime}$. The defect is just that this transform $V^{\prime}$ can possibly not be taken again for the subsequent blowups. In this situation, Abhyankar proposed, at least for plane curves, to change the hypersurface after each blowup if necessary. Again one gets a sequence of hypersurfaces, but they will no longer be related as transforms of each other under blowup. The descent becomes more complicated. Moreover, there is a priori no canonical choice for those hypersurfaces.

In the next section we shall describe this descent in more detail and explain how one can still formulate a resolution problem in smaller dimension. However, its solution is much harder, and up to now it has only been achieved for curves and surfaces.

D. Kangaroo phenomenon. Recall that the now classical resolution invariant in characteristic zero consists of a vector of integers whose components are orders of ideals in decreasing dimensions 9 The ideals are the consecutive coefficient ideals in hypersurfaces of maximal contact, and the vector is considered with respect to the lexicographic ordering. Two things are then shown: first, that the locus of points of $X$ where the invariant attains its maximal value is closed, smooth and transversal to the possibly already existing exceptional divisor (stemming from earlier blowups); and second, that the invariant drops under blowup when taking as center this locus of maximal values, as long as the ideals in lower dimension are not yet resolved (in a precise sense). The decrease allows us to apply induction (the lexicographic order is a well ordering) and to reduce by a finite sequence of blowups to the case where the invariant attains its minimal possible value. We arrive in this way at the so-called monomial case, for which an instant combinatorial description of the resolution is known. This program appears in different disguises in many places, see, e.g., Hi5, Vi1, BM, EV1, EH, W1, Ko,

In section $\mathrm{E}$ we will review the characteristic-free version of the characteristic zero invariant of an ideal at a point as it was developed in [Ha1, [EH]. For this definition, hypersurfaces of maximal contact (which need not exist in arbitrary characteristic) have to be replaced by hypersurfaces of weak maximal contact. These are defined as local smooth hypersurfaces that maximize the order of the coefficient ideal of the given ideal (as hypersurfaces of maximal contact do), but whose transforms, in contrast, are not required to contain the points along a sequence of blowups where the order of the original ideal remains constant.

Take then as resolution invariant the lexicographic vector consisting of the order of the ideal and of the orders of the iterated coefficient ideals with respect to such hypersurfaces. It turns out that the resulting vector (more precisely, its second component given by the order of the first coefficient ideal) may increase in positive characteristic under certain (permissible) blowups. The first examples of this phenomenon were observed by Abhyankar, Cossart, Moh and Seidenberg [Co, Mo, Se]. See also the work of Panazzolo on the resolution of real analytic vector fields in three variables $\mathrm{Pa}$. At first glance the increase destroys any kind of induction.

\footnotetext{
${ }^{9}$ For the basics on resolution in characteristic zero, you may consult [Ha3 Lp2, Ko].
} 

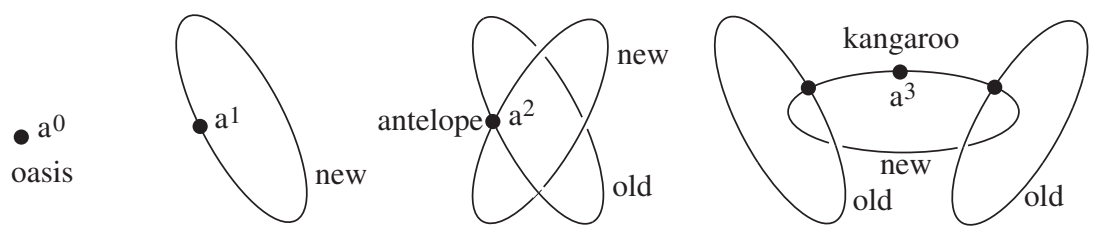

Figure 1. The configuration of kangaroo, antelope and oasis points.

Moh succeeded in bounding the maximal increase, but it was not yet possible to profit from this bound so as to save the induction argument (except for surfaces).

We shall describe accurately the situations in which an increase of the invariant occurs. To make the increase happen, the variety which is blown up must have a wild singularity. It is located at a so-called antelope point of the current stage of the sequence of blowups we are considering. On the transform of the variety, the increase of the invariant can then only occur at a kangaroo point 10 The location of these points and the structure of the singularities is meanwhile well understood and can be explained quite explicitly (cf. section G). Kangaroo points always lie on the new exceptional component of the last blowup but never on the transforms of the (old) exceptional components passing through the preceding antelope point (see Figure 1). This phenomenon is also known as the occurence of a "a translational blowup". To have a wild singularity at an antelope point preceding a kangaroo point, three conditions must hold: the residues modulo $p$ of the multiplicities of the exceptional components appearing in the defining equation must satisfy a certain arithmetic inequality, the order of the coefficient ideal of the equation must be $d i$ visible by the order of the equation, and strong restrictions on the (weighted) initial form of the defining equation are imposed (cf. the theorem in section $\mathrm{G}$ on kangaroo points). It turns out that the initial form of a wild singularity must be equal (up to multiplication by $p$ th powers) to an oblique polynomial. Oblique polynomials are characterized by a very peculiar behaviour under linear coordinate changes when considered up to addition of $p$ th powers. Fixing the exceptional multiplicities and the degree, both subject to the arithmetic and divisibility condition, it can be shown that there is precisely one oblique polynomial with these parameters (cf. section I).

For surfaces, it is possible to show that the characteristic zero resolution invariant decreases in the long run also in positive characteristic; i.e., that the occasional increases are compensated by decreases in the blowups before and after them. A first method for proving this was developed in [Ha1 and will be sketched in section $\mathrm{J}$ below. A second, more systematic approach introducing the bonus of a singularity appears in [HW]. It "adjusts" the characteristic zero invariant in the critical situations by a small correction value - the bonus - so as to ensure a permanent decrease of the invariant. We emphasize that there are earlier proofs of resolution of surfaces in arbitrary characteristic by Abhyankar, Lipman and Hironaka using different invariants and arguments [Ab1, Lp1, Hi4]. For three-folds and higher dimensional varieties, no complete induction argument for the embedded resolution seems to be known.

\footnotetext{
${ }^{10}$ In [Hi1], kangaroo points run under the name of metastatic points.
} 
With these remarks we conlude the general introduction. In the next sections, more detailed information will be given.

\section{Detailed Discussion}

E. The invariant. We define only the first two components of the classical resolution invariant as these suffice for the phenomena to be described here. For an ideal sheaf $\mathcal{J}$ on a smooth ambient space $W$ and a point $a \in W$ denote by $J=J_{a}$ the stalk of $\mathcal{J}$ at $a$. For convenience, we denote - if appropriate-by the same character $J$ the ideal generated in the completion $\widehat{\mathcal{O}}_{W, a}$ of the local ring $\mathcal{O}_{W, a} 11$ For a local smooth hypersurface $V$ in $W$ through $a$, the coefficient ideal of $J$ in $V$ is defined as the ideal

$$
\operatorname{coeff}_{V} J=\sum_{i=0}^{o-1}\left(a_{f, i}, f \in J\right)^{\frac{o !}{o-i}},
$$

where $o=\operatorname{ord}_{a} J$ is the order of $J$ at $a, x=0$ is a local equation for $V$ and $f=\sum a_{f, i} x^{i}$ is the expansion of $f$ with respect to $x$, with coefficients $a_{f, i} \in \mathcal{O}_{V, a}$. Among the many variants of this definition in the literature, the one given suits best our purposes. More specifications appear in $\mathrm{EH}$.

In case $J$ is a principal ideal generated by one polynomial $f(x, y)=x^{o}+g(y)$ in $\mathbb{A}^{1+m}$ with variables $x$ and $y=\left(y_{m}, \ldots, y_{1}\right)$, the coefficient ideal of $J$ with respect to the hypersurface $x=0$ is simply the ideal in $\mathbb{A}^{m}$ generated by $g^{(o-1) !}$. The factorial is only needed to ensure integer exponents when $f$ has other $x$ terms.

The order of the coefficient ideal at $a$ depends on the choice of the hypersurface $V$, but remains unchanged under passing to the completions of the local rings. The supremum of these orders over all choices of local smooth hypersurfaces $V$ through $a$ is a local invariant of $J$ at $a$ (i.e., by definition, it only depends on the isomorphism class of the complete local ring $\left.\widehat{\mathcal{O}}_{W, a} / J\right)$. This supremum is $\infty$ if and only if $J$ is bold regular at $a$, viz generated by a power of a parameter of $\widehat{\mathcal{O}}_{W, a}[\mathrm{EH}]$. If the supremum is finite and hence a maximum, any hypersurface $V$ realizing this value is said to have weak maximal contact with $J$ at $a$. In characteristic zero, hypersurfaces of maximal contact have weak maximal contact [EH]. Moreover, their strict transforms under a permissible blowup $W^{\prime} \rightarrow W$ contain all equiconstant points (classically called infinitely near points in $W^{\prime}$ ), i.e., those points of the exceptional divisor where the order of the weak transform $J^{\prime}$ of $J$ has remained constant (recall that this order cannot increase if $J$ has constant order along the center).

In arbitrary characteristic, the supremum of the orders of the coefficient ideal coeff $_{V} J$ for varying $V$ can be used to define the second component of the candidate resolution invariant of $J$ at $a$. If the supremum is $\infty$ and thus $J$ is bold regular, a resolution is already achieved locally at $a$, so we discard this case. We henceforth assume that the supremum is finite and can thus be realized by the choice of a suitable hypersurface $V$. After factoring a suitable divisor from the resulting coefficient ideal, one takes the order of the remaining factor as the second component of the invariant. More explicitly, let $D$ be a given normal crossings divisor in $W$ with defining ideal $I_{W}(D)$. We shall assume throughout that coeff $V_{V} J$ factors for any chosen $V$ transversal to $D$ (in the sense of normal crossings) into a product of

\footnotetext{
${ }^{11}$ You may think here that $\mathcal{J}$ is an ideal in a polynomial ring and $J$ is the induced ideal in a formal power series ring generated by the Taylor expansions of the elements of $\mathcal{J}$ at a point.
} 
ideals

$$
\operatorname{coeff}_{V} J=I_{V}(D \cap V) \cdot I_{-},
$$

where $I_{-}$is some ideal in $\widehat{\mathcal{O}}_{V, a}$ (this assumption is always realized in practice). Then define the shade of $J$ at a with respect to $D$ as the maximum value $\operatorname{shade}_{a} J$ of $\operatorname{ord}_{a} I_{-}$over all choices of $V$ transversal to $D$. In Hi1, a similarly defined invariant is considered by Hironaka and is called the residual order of $J$ at a. As usual, questions of well-definedness and upper-semicontinuity have to be taken care of 12

Along a resolution process, $D$ will always be supported by the exceptional components accumulated so far. It coincides with the second entry of the combinatorial handicap of a mobile as defined in [EH]. At the beginning, or whenever $\operatorname{ord}_{a} J$ has dropped, $D$ will be empty. If the order of $J$ has remained constant at a point $a^{\prime}$ above $a$, the transform $D^{\prime}$ of $D$ is defined as

$$
D^{\prime}=D^{\curlyvee}+\left(\operatorname{ord}_{a}(D \cap V)+\operatorname{shade}_{a} J-\operatorname{ord}_{a} J\right) \cdot Y^{\prime},
$$

where $Y^{\prime}$ denotes the exceptional divisor of the last blowup, and $D^{r}$ the strict transform of $D 13$ The formula signifies that $D^{\prime}$ consists of the transform of $D$ together with the new exceptional component $Y^{\prime}$ (which is taken with a suitable multiplicity). Note that $\operatorname{ord}_{a}(D \cap V)+\operatorname{shade}_{a} J=\operatorname{ord}_{a}\left(\operatorname{coeff}_{V} J\right)$. It follows from the transformation rule of $D$ that, under permissible blowup, the weak transform $J^{\prime}$ of $J$ at an equiconstant point $a^{\prime}$ above $a$ has as coefficient ideal coeff $V_{V^{\prime}} J^{\prime}$ in the strict transform $V^{\prime}$ of $V$ an ideal which factors again into a product $I_{V^{\prime}}\left(D^{\prime} \cap V^{\prime}\right) \cdot I_{-}^{\prime}$, with $I_{-}^{\prime}$ the weak transform $\left(I_{-}\right)^{\gamma}$ of $I_{-}$. Here, it is assumed that the center $Z$ is contained in $V$. This is more delicate to achieve in positive characteristic, due to the example of Narasimhan where the singular locus of $J$ is not contained locally in any smooth hypersurface [Na1, $\mathrm{Na2}, \mathrm{Mu}$. It can, however, be realized by refining the usual stratification of the singular locus of $J$ through the local embedding dimension of this locus.

We say that the monomial case occurs when the whole coefficient ideal has become an exceptional monomial, say $\operatorname{coeff}_{V} J=I_{V}(D \cap V)$ with $I_{-}=1$. The shade has then attained its minimal value 0 . This case allows a purely combinatorial resolution of $J$.

The commutativity of the passage to coefficient ideals with blowups can be subsumed as follows (cf. [EH, Ha2]). Given a blowup with center $Z$ contained in the local hypersurface $V$ of $W$ locally at $a$ and transversal to $D$, we get for any equiconstant $a^{\prime}$ in $W^{\prime}$ above $a$ and $I_{-}^{\prime}=\left(I_{-}\right)^{\curlyvee}$ a commutative diagram

$$
\begin{array}{ccccc}
J^{\prime} & \rightsquigarrow \operatorname{coeff}_{V^{\prime}} J^{\prime} & = & I_{V^{\prime}}\left(D^{\prime} \cap V^{\prime}\right) \cdot I_{-}^{\prime} \\
\downarrow & & \downarrow & & \\
J & \operatorname{coeff}_{V} J & = & I_{V}(D \cap V) \cdot I_{-}
\end{array}
$$

Here, the situation splits according to the characteristic: in characteristic zero, choosing for $V$ a hypersurface of maximal contact for $J$ at $a$, the strict transform $V^{\prime}$ constitutes again a hypersurface of maximal contact for $J^{\prime}$ at $a^{\prime}$. In particular, both will have weak maximal contact so that the shades of $J$ and $J^{\prime}$ are well defined. In addition, shade $_{a^{\prime}} J^{\prime}$ can be computed from shade ${ }_{a} J$ by looking at the blowup

\footnotetext{
${ }^{12}$ Semicontinuity works well if only closed points are considered. For arbitrary (i.e., nonclosed) points, there appear pathologies which are described and studied by Hironaka Hi1].

${ }^{13}$ We use here implicitly that $V$ and $Z$ are transversal to $D$. This is indeed the case in the resolution process of an ideal or scheme.
} 
$V^{\prime} \rightarrow V$ with center $Z$ and the ideals $I_{-}$and $I_{-}^{\prime}$ (recall that $Z \subset V$ locally at a). As $\operatorname{shade}_{a} J=\operatorname{ord}_{a} I_{-}, \operatorname{shade}_{a^{\prime}} J^{\prime}=\operatorname{ord}_{a^{\prime}} I_{-}^{\prime}$ and $I_{-}^{\prime}$ is the weak transform of $I_{-}$, it follows automatically that $\operatorname{shade}_{a^{\prime}} J^{\prime} \leq \operatorname{shade}_{a} J$ (it is required here that the order of $I_{-}$is constant along $Z$, a property that is achieved through the insertion of companion ideals as suggested by Villamayor, cf. [EV2, EH]). This makes the induction and the descent in dimension work.

In positive characteristic, it is in general not possible to choose a local hypersurface of maximal contact for $J$ at $a$. But a hypersurface of weak maximal contact will always exist, by definition. So choose one, say $V$. The good news is, as Zariski already observed $\left[\mathrm{Za}\right.$, that the strict transform $V^{\prime}$ of $V$ will contain all equiconstant points $a^{\prime}$ of $J$ in the exceptional divisor $Y^{\prime}$. The bad news is, as Moh's and Narasimhan's examples show, that $V^{\prime}$ need no longer have weak maximal contact with $J^{\prime}$ at $a^{\prime}$. Said differently, $V^{\prime}$ need not maximize the order of the coefficient ideal of the weak transform $J^{\prime}$ of $J$ at $a^{\prime}$. One may have to choose a new hypersurface $U^{\prime}$ at $a^{\prime}$ to maximize this order. As Moh observed [Mo], there is still worse news, since the choice of $U^{\prime}$ may produce a shade of $J^{\prime}$ at $a^{\prime}$ which is strictly larger than the shade of $J$ at $a$. This destroys the induction over the lexicographically ordered pair $\left.\operatorname{ord}_{a}(J), \operatorname{shade}_{a}(J)\right)$. At least at first sight!

F. Moh's bound. In his paper on local uniformization, Moh investigates the possible increase of $\operatorname{shade}_{a} J$ at equiconstant points $a^{\prime}$ of $J$ in the purely inseparable case

$$
f(x, y)=x^{p^{e}}+y^{r} \cdot g(y),
$$

with $\operatorname{ord}\left(y^{r} g\right) \geq p^{e}=\operatorname{ord} f$ and $e \geq 1$ [Mo, 14 Here, $V$ defined by $x=x_{n}=0$ denotes a hypersurface of weak maximal contact for $f$ at $a=0$ in $W=\mathbb{A}^{n}, p$ is the characteristic of the (algebraically closed) ground field, and $y=\left(x_{n-1}, \ldots, x_{1}\right)$ denote further coordinates so that $(x, y)$ form a complete parameter system of $R=$ $\widehat{\mathcal{O}}_{\mathbb{A}^{n}, 0}$. Moreover, $r \in \mathbb{N}^{n-1}$ is a multiexponent whose entries are the multiplicities of the components of the divisor $D \cap V$ at 0 , and $y_{i}=0$ defines an irreducible component of $D \cap V$ in $V$ for all $i$ for which $r_{i}>0$. All expressions take place in the algebra of an étale neighborhood of 0 in $\mathbb{A}^{n}$, so that $f$ and possible coordinate changes are considered as formal power series. The shade of $f$ at 0 with respect to the divisor $D$ defined by $y^{r}=0$ is given by $\operatorname{shade}_{0} f=\operatorname{ord}_{0} g$, by the choice of $V$.

Proposition (Moh). In the above situation, let $\left(W^{\prime}, a^{\prime}\right) \rightarrow(W, a)$ be a local blowup with smooth center $Z$ contained in the top locus of $f$ and transversal to $D$. Assume that $a^{\prime}$ is an equiconstant point for $f$ at a, i.e., $\operatorname{ord}_{a^{\prime}} f^{\prime}=\operatorname{ord}_{a} f=p^{e}$, where $f^{\prime}$ denotes the strict transform of $f$ at $a^{\prime}$. Then

$$
\operatorname{shade}_{a^{\prime}} f^{\prime} \leq \operatorname{shade}_{a} f+p^{e-1} .
$$

In case $e=1$, the inequality reads $\operatorname{shade}_{a^{\prime}} f^{\prime} \leq \operatorname{shade}_{a} f+1$, which is not too bad, but still unpleasant. The short proof of Moh uses a nice trick with derivations, thus eliminating all $p$ th powers from $y^{r} g(y)$. He then briefly investigates the case where an increase of the shade indeed occurs, showing that in the next blowup the shade has to drop at least by 1 (if $e=1$ ). This, obviously, does not suffice yet to make induction work.

\footnotetext{
${ }^{14}$ It seems that Abhyankar had already observed this increase.
} 
G. Kangaroo points and wild singularities. In the following paragraphs we reproduce in compact form the classification of kangaroo points and wild singularities given in Ha1. Recall: The shade of a polynomial $f$ at a point $a$ with respect to a normal crossings divisor $D$ is the maximal value of the order of its coefficient ideal minus the multiplicity of $D$ at $a$, the maximum being taken over all choices of smooth local hypersurfaces at $a$ transversal to $D$. A kangaroo point in a blowup $W^{\prime} \rightarrow W$ with permissible center $Z$ and exceptional divisor $Y^{\prime}$ is an equiconstant point $a^{\prime}$ above $a \in Z$ where the shade of $f$ with respect to $D$ has increased,

$$
\operatorname{ord}_{a^{\prime}} f^{\prime}=\operatorname{ord}_{a} f \quad \text { and } \text { shade }_{a^{\prime}} f^{\prime}>\text { shade }_{a} f .
$$

Here, shade $a^{\prime} f^{\prime}$ is taken with respect to the divisor

$$
D^{\prime}=D^{\curlyvee}+\left(\operatorname{ord}_{a}(D \cap V)+\operatorname{shade}_{a} f-\operatorname{ord}_{a} f\right) \cdot Y^{\prime},
$$

where $D^{\curlyvee}$ denotes the strict transform of $D$ and $\operatorname{ord}_{a} f=\operatorname{ord}_{Z} f$ holds by permissibility of $Z$. The point $a$ prior to a kangaroo point $a^{\prime}$ is called an antelope point. Note here that if $V$ and $V^{\prime}$ are hypersurfaces of weak maximal contact, then $\operatorname{ord}_{a}(D \cap V)=\operatorname{ord}_{a} D$ and $\operatorname{ord}_{a^{\prime}}\left(D^{\prime} \cap V^{\prime}\right)=\operatorname{ord}_{a^{\prime}} D^{\prime}$ by transversality of $D$ and $D^{\prime}$ with $V$ and $V^{\prime}$.

For the ease of the exposition, we restrict our discussion to hypersurfaces in $\mathbb{A}^{n}=\mathbb{A}^{1+m}$ with purely inseparable equation $f(x, y)=x^{p}+y^{r} \cdot g(y)$ of order $p$ at 0 equal to the characteristic, with exceptional multiplicities $r=\left(r_{m}, \ldots, r_{1}\right) \in \mathbb{N}^{m}$ and coordinates $(x, y)=\left(x, y_{m}, \ldots, y_{1}\right)$. We shall work only at closed points and with formal power series. Moreover, we confine to point blowups, since these entail the most delicate problems. Most of the concepts and results go through for more general situations. For an integral vector $r \in \mathbb{N}^{m}$ and a number $c \in \mathbb{N}$, let $\phi_{c}(r)$ denote the number of components of $r$ that are not divisible by $c$,

$$
\phi_{c}(r)=\#\left\{i \leq m, r_{i} \not \equiv 0 \bmod c\right\} .
$$

Define $\bar{r}^{c}=\left(\bar{r}_{m}^{c}, \ldots, \bar{r}_{1}^{c}\right)$ as the vector of the residues $0 \leq \bar{r}_{i}^{c}<c$ of the components of $r$ modulo $c$, and let $|r|=r_{m}+\cdots+r_{1}$.

The next theorem characterizes kangaroo points and wild singularities. We state it here only in its simplest form for purely inseparable equations of order $p$ at 0 . An appropriate extension also holds beyond the purely inseparable case for singularities of any order and for blowups in positive dimensional centers; see section $\mathrm{H}$ below, respectively Ha1, Thm. 1, sec. 5, and Thm. 2, sec. 12] and its forthcoming update Ha2.

Kangaroo Theorem (Hauser). Let $\left(W^{\prime}, a^{\prime}\right) \rightarrow(W, a)$ be a local point blowup of $W=\mathbb{A}^{1+m}$ with center $Z=\{a\}=\{0\}$ and exceptional divisor $Y^{\prime}$. Let $X$ be a hypersurface singularity in $W$ at a with equation $f=0$. Let there be given local coordinates $\left(x, y_{m}, \ldots, y_{1}\right)$ at a so that $f(x, y)=x^{p}+y^{r} \cdot g(y)$ has order $p$ and shade $\operatorname{ord}_{a} g$ with respect to the divisor $D$ defined by $y^{r}=0$. Then, for a to be a wild singularity of $X$ with kangaroo point $a^{\prime}$, the following conditions must hold at $a$ :

(1) The order $|r|+\operatorname{ord}_{a} g$ of $y^{r} g(y)$ is a multiple of $p$.

(2) The exceptional multiplicities $r_{i}$ at a satisfy

$$
\bar{r}_{m}^{p}+\cdots+\bar{r}_{1}^{p} \leq\left(\phi_{p}(r)-1\right) \cdot p .
$$


(3) The location of $a^{\prime}$ on $Y^{\prime}$ is determined by the expansion of $f$ at a. It lies on none of the strict transforms of the exceptional components $y_{i}=0$ for which $r_{i}$ is not a multiple of $p$.

(4) The initial form of $g$ equals, up to linear coordinate changes and multiplication by pth powers, a specific homogeneous polynomial, called oblique, which is unique for each choice of $p, r$ and degree 15

Remarks. (a) The above characterization seems to have been a vital step in Hironaka's recent approach to the resolution of singularities in positive characteristic (cf. [Hi1, Prop. 13.1 and Thm. 13.2]).

(b) The necessity of condition (1) is easy to see and already appears in $\mathrm{Mo}$. The arithmetic inequality in condition (2) is related to counting the number of $p$-multiples in simplices in $\mathbb{R}^{m}$ and their $r$-translates 16 It implies that at least two exponents $r_{i}$ must be prime to $p$. For surfaces $(m=2)$, condition (2) reads $r_{2}, r_{1} \not \equiv 0 \bmod p$ and $\bar{r}_{2}+\bar{r}_{1} \leq p$. Condition (3) implies that the reference point has to jump off from all exceptional components with $r_{i} \not \equiv 0 \bmod p$ in order to arrive at a kangaroo point. So it has to leave at least two exceptional components (cf. Figure 1 from the Introduction). This, together with the jump of $\operatorname{shade}_{a} f$, justifies the naming of these points. Condition (4) will be discussed in the example below and in section I on oblique polynomials.

(c) Conditions (1) to (4) are necessary for the occurrence of kangaroo points. They are also sufficient, up to the higher degree terms of $g$, in the following sense: In the transform $g^{\prime}$ of $g$ the terms of $g$ of degree $>\operatorname{ord}_{a} g$ (i.e., not in the initial form) may have transformed into terms of degree smaller than the order of the transform $\bar{g}^{\prime}$ of the initial form $\bar{g}$ of $g$. This signifies that $\operatorname{ord}_{a^{\prime}} g^{\prime}<\operatorname{ord}_{a^{\prime}} \bar{g}^{\prime}$. As $\operatorname{ord}_{a^{\prime}} g^{\prime}=\operatorname{shade}_{a^{\prime}} f^{\prime}, \operatorname{ord}_{a} g=\operatorname{shade}_{a} f$ by definition, and $\operatorname{ord}_{a^{\prime}} \bar{g}^{\prime} \leq \operatorname{shade}_{a} \bar{f}+1=$ shade $_{a} f+1$ with $\bar{f}=x^{p}+y^{r} \bar{g}$ by Moh's bound applied to $\bar{f}$, the strict inequality shade $_{a^{\prime}} f^{\prime}>$ shade $_{a} f$ becomes impossible. The influence of the higher order terms of $g$ can be made quite explicit in concrete examples.

(d) We emphasize that the intricacy of the resolution in positive characteristic lies precisely in these higher order terms. Without them, $g$ is homogeneous (and thus equal to its initial form). In this case it is easy to make the order of $f$ drop below $p$ by suitable further blowups. But, in the general case, it seems to be tricky to control $g$ beyond its initial form.

Example 3. For surfaces $(n=3$ and $m=2)$, condition (2) reads $\bar{r}_{2}+\bar{r}_{1} \leq p$, provided that $r_{2}, r_{1}>0$. In this case, there is an explicit description of the initial form $P=\bar{g}$ of $g$ as indicated by condition (4): If $\left(\begin{array}{c}k+r \\ k+1\end{array}\right)$ is not a multiple of $p$ it has the form

$$
P(y, z)=y^{r} z^{s} \cdot \mathbb{H}_{r}^{k}(y, t z-y)=y^{r} z^{s} \cdot \sum_{i=0}^{k}\left(\begin{array}{c}
k+r \\
i+r
\end{array}\right) y^{i}(t z-y)^{k-i},
$$

where $r=r_{1}, s=r_{2}, k=\operatorname{ord}_{a} g$, and $t$ is some nonzero constant in the ground field. The constant $t$ determines the location of $a^{\prime}$ on the exceptional divisor $Y^{\prime}$,

\footnotetext{
${ }^{15}$ The possibility of multiplication with $p$ th powers was not properly indicated in the original version of Ha1] (though it followed from the proof given there).

${ }^{16}$ The inequality is equivalent to $\left\lceil\frac{\bar{r}_{1}^{p}}{p}\right\rceil+\cdots+\left\lceil\frac{\bar{r}_{m}^{p}}{p}\right\rceil>\left\lceil\frac{\bar{r}_{1}^{p}+\cdots+\bar{r}_{m}^{p}}{p}\right\rceil$, where $\lceil u\rceil$ is the smallest integer $\geq u$. In this case the simplex $\Delta=\left\{\alpha \in \mathbb{N}^{m},|\alpha|=\operatorname{ord}_{a} g\right\}$ contains more $p$-multiples than its translate $r+\Delta$.
} 
and vice versa. The polynomials

$$
\mathbb{H}_{r}^{k}(y, w)=\sum_{i=0}^{k}\left(\begin{array}{c}
k+r \\
i+r
\end{array}\right) y^{i} w^{k-i}
$$

are called hybrid polynomials of type $(r, k)$ in [Ha1. Note that we can write $\mathbb{H}_{r}^{k}$ as

$$
\begin{aligned}
\mathbb{H}_{r}^{k}(y, w) & =\sum_{i=0}^{k}\left(\begin{array}{c}
k+r \\
k-i
\end{array}\right) y^{i} w^{k-i} \\
& =\sum_{i=0}^{k}\left(\begin{array}{c}
k+r \\
i
\end{array}\right) y^{k-i} w^{i} \\
& =y^{-r} \cdot \sum_{i=0}^{k}\left(\begin{array}{c}
k+r \\
i
\end{array}\right) y^{k+r-i} w^{i} \\
& =\left\lfloor y^{-r} \cdot \sum_{i=0}^{k+r}\left(\begin{array}{c}
k+r \\
i
\end{array}\right) y^{k+r-i} w^{i}\right\rfloor_{\text {poly }} \\
& =\left\lfloor y^{-r} \cdot(y+w)^{k+r}\right\rfloor_{\text {poly }},
\end{aligned}
$$

where $\lfloor Q\rfloor_{\text {poly }}$ denotes those terms of the Laurent expansion of $Q$ that involve no monomials with negative exponents.

Rocío Blanco observed that if $\left(\begin{array}{c}k+r \\ k+1\end{array}\right)$ is a multiple of $p$, the above polynomial $P=y^{r} z^{s} \cdot \mathbb{H}_{r}^{k}(y, t z-y)$ is a $p$ th power and thus does not count. In this case one can use alternatively a description of the initial form of $g$ which is independent of the divisibility of $\left(\begin{array}{c}k+r \\ k+1\end{array}\right)$ by $p$ (cf. section I below):

$$
\begin{aligned}
P(y, z) & =z^{s} \cdot \int y^{r-1}(y-t z)^{k} d y \\
& =z^{s} \cdot \sum_{i=0}^{k}(-1)^{k-i} \frac{1}{r+i} y^{r+i}(t z)^{k-i},
\end{aligned}
$$

the sum being taken over those $i$ for which $r+i$ is not divisible by $p$. Dominique Wagner showed that the two formulas for $P$ differ - up to adding $p$ th powers - by the scalar factor $(-1)^{k}\left(\begin{array}{c}k+r \\ k+1\end{array}\right)(k+1)$. This explains why the first formula requires that $\left(\begin{array}{l}k+r \\ k+1\end{array}\right)$ is prime to $p$.

Let us illustrate the dependence on $p$ in the case $p=k=2, r=s=2$, where the binomial coefficient $\left(\begin{array}{l}k+r \\ k+1\end{array}\right)=\left(\begin{array}{l}5 \\ 3\end{array}\right)=10$ is not prime to $p$. Indeed,

$$
\begin{aligned}
y^{r} z^{s} \cdot & \mathbb{H}_{r}^{k}(y, t z-y) \\
& =y^{3} z^{3} \cdot\left[\left(\begin{array}{l}
5 \\
3
\end{array}\right)(t z-y)^{2}+\left(\begin{array}{l}
5 \\
4
\end{array}\right) y(t z-y)+\left(\begin{array}{l}
5 \\
5
\end{array}\right) y^{2}\right] \\
& =y^{3} z^{3} \cdot\left[10(t z-y)^{2}+5 y(t z-y)+y^{2}\right] \\
& =y^{3} z^{3} \cdot\left[y(t z-y)+y^{2}\right] \\
=t & y^{4} z^{4}
\end{aligned}
$$


is a $p$ th power (provided that $K$ is perfect) and thus does not count as oblique, whereas

$$
\begin{aligned}
P(y, z) & =z^{s} \cdot \int y^{r-1}(y-t z)^{k} d y \\
& =z^{3} \cdot \int y^{2}(y-t z)^{2} d y \\
& =z^{3} \cdot \int\left(y^{4}+t^{2} y^{2} z^{2}\right) d y \\
& =y^{3} z^{3} \cdot\left(y^{2}+t^{2} z^{2}\right)
\end{aligned}
$$

produces an increase of the shade. In section I, we characterize oblique polynomials in arbitrary dimension.

H. Proof of the Kangaroo Theorem. We indicate the main points of the argument for arbitrary polynomials $f$, i.e., in the case where $f$ is not necessarily purely inseparable. This makes things more complicated but has the advantage of being generally applicable in a resolution process. The argument should be compared with the (much simpler) computation of oblique polynomials for the purely inseparable case which is given in section I. Along the way, one obtains an alternative proof of Moh's inequality.

It is convenient to work in the power series ring and to assume that $f$ is in Weierstrass form of order, say $c$, with respect to the variable $x$. It then suffices to consider a weighted homogeneous $f$ with respect to weights $(w, 1, \ldots, 1)$ where $w \geq 1$ is the ratio between the order of $f$ and the order of its coefficient ideal with respect to $x$, say $w=$ ord $f /$ ord $\operatorname{coeff}_{x}(f)$.

The inequality $\bar{r}_{m}^{c}+\cdots+\bar{r}_{1}^{c} \leq\left(\phi_{c}(r)-1\right) \cdot c$ allows us to count the lattice points that lie in certain integral simplices in $\mathbb{R}_{+}^{n}$ (called zwickels in [Ha1]) but do not belong to the sublattice $p \cdot \mathbb{Z}^{n}$. The key step of the proof of the Kangaroo Theorem is then to establish the invertibility of the transformation matrix between the vectors of coefficients of polynomials with exponents in such zwickels under prescribed coordinate changes, the polynomials being always considered modulo $p$ th powers. For illustration, we reproduce the corresponding passage from section 11 of $\mathrm{Ha1}$.

Let $f(x, y)$ and $\tilde{f}(x, y)=f\left(x+\sum_{\gamma} h_{\gamma} y^{\gamma}, y+t y_{m}\right)$ be weighted homogeneous polynomials of weighted degree $e$ with respect to weights $(w, 1, \ldots, 1)$ on $(x, y)=\left(x, y_{m}, \ldots, y_{1}\right)$, where the sum $\sum_{\gamma} h_{\gamma} y^{\gamma}$ ranges over $\gamma \in \mathbb{N}^{m}$ with $|\gamma|=w$, and where $h_{\gamma}$ and the components of $t=\left(0, t_{m-1}, \ldots, t_{1}\right)$ belong to the ground field. Let $c=e / w$ be the order of $f$. Write

$$
f(x, y)=\sum a_{k \alpha} x^{k} y^{\alpha} \quad \text { and } \quad \tilde{f}(x, y)=\sum b_{l \beta}(t) x^{l} y^{\beta}
$$

with $w k+|\alpha|=w l+|\beta|=e$. We assume that $a_{c 0} \neq 0$, i.e., that $x^{c}$ appears with nonzero coefficient, say $a_{c 0}=1$. Let $V$ be the hypersurface in $W=\mathbb{A}^{n}$ defined by $x=0$. Let

$$
L_{c}=\left\{(k, \alpha) \in \mathbb{N}^{1+m}, k<c\right\} \rightarrow \mathbb{Q}^{m}:(k, \alpha) \rightarrow \frac{c}{c-k} \cdot \alpha
$$

be the map projecting elements $(k, \alpha)$ of the layer $L_{c}$ in $\mathbb{N}^{1+m}$ to elements of $\mathbb{Q}^{m}$. The center of the projection is the point $(c, 0, \ldots, 0)$. 
Let $q \in \mathbb{N}^{m}$ with $|q|=q_{m}+\cdots+q_{1} \leq e$ be fixed. Define the upper zwickel $Z(q)$ in $\mathbb{N}^{1+m}$ as the set of points $(k, \alpha)$ with $0 \leq k \leq c$, $w k+|\alpha|=e$ and projection $\frac{c}{c-k} \cdot \alpha \geq_{c p} q$, denoting by $\geq_{c p}$ the componentwise order. Thus $Z(q)$ is given by

$$
Z(q): w k+|\alpha|=e \quad \text { and } \quad \alpha \geq_{c p}\left\lceil\frac{c-k}{c} \cdot\left(q_{m}, \ldots, q_{1}\right)\right\rceil .
$$

Let us fix a decomposition $q=r+\ell \in \mathbb{N}^{m}$ with $r=\left(q_{m}, \ldots, q_{j+1}\right.$, $0, \ldots, 0)$ and $\ell=\left(0, \ldots, 0, q_{j}, \ldots, q_{1}\right)$ for some index $j$ between $m-1$ and 0 . Define the lower zwickel $Y(r, \ell)$ in $\mathbb{N}^{1+m}$ as the set of points $(k, \beta)$ in $\mathbb{N}^{1+m}$ with $0 \leq k \leq c, w k+|\beta|=e$ and projection $\frac{c}{c-k} \cdot \beta \geq_{c p}(|r|, 0, \ldots, 0, \ell)$. Thus $Y(r, \ell)$ is given by

$Y(r, \ell): w k+|\beta|=e \quad$ and $\quad \beta \geq_{c p}\left\lceil\left(\frac{c-k}{c} \cdot|r|, 0, \ldots, 0, \frac{c-k}{c} \cdot q_{j}, \ldots, \frac{c-k}{c} \cdot q_{1}\right)\right\rceil$.

For $j=m-1$ and hence $r=\left(q_{m}, 0, \ldots, 0\right)$ and $\ell=\left(0, q_{m-1}, \ldots, q_{1}\right)$ we have $Z(q)=Y(r, \ell)$. In general, the two zwickels are different.

For any $r$ and $\ell$ and $0 \leq k \leq e / w=c$, the slice

$$
Y(r, \ell)(k)=\{(k, \beta) \in Y(r, \ell)\}=Y(r, \ell) \cap\left(\{k\} \times \mathbb{N}^{m}\right)
$$

has at least as many elements as the slice

$$
Z(q)(k)=\{(k, \alpha) \in Z(q)\}=Z(q) \cap\left(\{k\} \times \mathbb{N}^{m}\right) .
$$

This holds for $k=0$, by definition of $Z(q)$ and $Y(r, \ell)$. For arbitrary $k$, the inequality $\left\lceil\frac{c-k}{c} \cdot|r|\right\rceil \leq\left|\left\lceil\frac{c-k}{c} \cdot r\right\rceil\right|$ implies that the condition

$$
w k+|\beta|=e \quad \text { and } \beta \geq_{c p}\left(\left|\left\lceil\frac{c-k}{c} \cdot r\right\rceil\right|, 0, \ldots, 0,\left\lceil\frac{c-k}{c} \cdot q_{j}\right\rceil, \ldots,\left\lceil\frac{c-k}{c} \cdot q_{1}\right\rceil\right)
$$

is more restrictive than the condition

$$
w k+|\beta|=e \quad \text { and } \beta \geq_{c p}\left(\left\lceil\frac{c-k}{c} \cdot|r|\right\rceil, 0, \ldots, 0,\left\lceil\frac{c-k}{c} \cdot q_{j}\right\rceil, \ldots,\left\lceil\frac{c-k}{c} \cdot q_{1}\right\rceil\right)
$$

defining $Y(r, \ell)(k)$. For each $k$, the set of pairs $k, \beta$ satisfying the first condition has as many elements as $Z(q)(k)$ because $|r|+q_{j}+$ $\cdots+q_{1}=|q|$. The claim follows.

It is immediate that $y^{q}$ is a factor of $\operatorname{coeff}_{V} f$ if and only if $f-x^{c}$ has all exponents in the upper zwickel $Z=Z(q)$ with $q \in \mathbb{N}^{m}$, and $\operatorname{coeff}_{V} \tilde{f}$ has order $>e-|r|$ in $z=\left(y_{m-1}, \ldots, y_{1}\right)$ if and only if all coefficients of the monomials of $\tilde{f}-x^{c}$ with exponent in the lower zwickel $Y(r, \ell)$ are zero.

Write elements $\beta \in \mathbb{N}^{m}$ as $\left(\beta_{m}, \beta^{-}\right)$where $\beta^{-}=\left(\beta_{m-1}, \ldots, \beta_{1}\right) \in$ $\mathbb{N}^{m-1}$. Let $Y^{*}(r, \ell)$ be the subset of $Y(r, \ell)$ of elements $(k, \beta) \in$ $\mathbb{N}^{1+m}$ given by

$$
\begin{aligned}
& \left|\beta^{-}\right| \leq e-w k-\left\lceil\frac{c-k}{c} \cdot|r|\right\rceil, \\
& \beta^{-} \geq_{c p}\left\lceil\frac{c-k}{c} \cdot\left(0, \ldots, 0, q_{j}, \ldots, q_{1}\right)\right\rceil .
\end{aligned}
$$

By definition, for each $k$, the slice $Y^{*}(r, \ell)(k)$ has the same cardinality as the slice $Z(q)(k)$ of the upper zwickel $Z(q)$. For $\alpha$ and $\delta$ in $\mathbb{Z}^{m}$, set $\left(\begin{array}{l}\alpha \\ \delta\end{array}\right)=\prod_{i}\left(\begin{array}{c}\alpha_{i} \\ \delta_{i}\end{array}\right)$, where $\left(\begin{array}{c}\alpha_{i} \\ \delta_{i}\end{array}\right)$ is zero if $\alpha_{i}<\delta_{i}$ or $\delta_{i}<0$. 
For $\Gamma$ a subset of $\mathbb{N}^{m}$, define for $k \in \mathbb{N}$ and $\lambda=\left(\lambda_{\gamma}\right)_{\gamma \in \Gamma} \in \mathbb{N}^{\Gamma}$ the alternate binomial coefficient

$$
\left[\left(\begin{array}{l}
k \\
\lambda
\end{array}\right)\right]=\prod_{\gamma \in \Gamma}\left(\begin{array}{c}
k-|\lambda|^{\gamma} \\
\lambda_{\gamma}
\end{array}\right) \quad \text { with }|\lambda|^{\gamma}=\sum_{\varepsilon \in \Gamma, \varepsilon<l_{\text {lex }} \gamma} \lambda_{\varepsilon} .
$$

Let $\Gamma \subset \mathbb{N}^{m}$ be the set of $\gamma \in \mathbb{N}^{m}$ with $|\gamma|=w$ and write $h=$

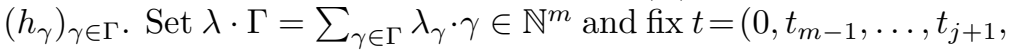
$0, \ldots, 0)$. We then have (cf. [Ha1, Prop. 1, sec. 11]) the following proposition.

Proposition. Let

$$
f(x, y)=\sum a_{k \alpha} x^{k} y^{\alpha}
$$

and

$$
\tilde{f}(x, y)=f\left(x+\sum_{\gamma \in \Gamma} h_{\gamma} y^{\gamma}, y+t y_{m}\right)=\sum b_{l \beta}(t) x^{l} y^{\beta}
$$

be weighted homogeneous polynomials with respect to weights $(w, 1, \ldots, 1)$ as above. Fix $q=r+\ell \in \mathbb{N}^{m}$ with zwickels $Z(q)$ and $Y^{*}(r, \ell) \subset Y(r, \ell)$.

(1) The transformation matrix $A=\left(A_{k \alpha, l \beta}\right)$ from the coefficients $a_{k \alpha}$ of $f$ to the coefficients $b_{l \beta}(t)$ of $\tilde{f}$ is given by

$$
A_{k \alpha, l \beta}=\sum_{\lambda \in \mathbb{N}^{\Gamma},|\lambda|=k-l}\left(\begin{array}{c}
k \\
l
\end{array}\right)\left[\left(\begin{array}{c}
k-l \\
\lambda
\end{array}\right)\right]\left(\begin{array}{c}
\alpha \\
\delta_{\alpha \beta \lambda}
\end{array}\right) \cdot h^{\lambda} \cdot t^{\alpha-\delta_{\alpha \beta \lambda}},
$$

where $\delta_{\alpha \beta \lambda}=\left(\alpha_{m}, \beta^{-}-(\lambda \cdot \Gamma)^{-}\right) \in \mathbb{N}^{m}$ and $h^{\gamma}=\Pi_{\gamma} h_{\gamma}^{\lambda_{\gamma}}$.

(2) The quadratic submatrix $A^{\square}=\left(A_{k \alpha, l \beta}\right)$ of $A$ with $(k \alpha, l \beta)$ ranging in $Z(q) \times Y^{*}(r, \ell)$ has determinant $t^{\rho\left(Z, Y^{*}(r, \ell)\right)}$ where $\rho\left(Z, Y^{*}(r, \ell)\right)$ is a vector in $\mathbb{N}^{m-1}$ independent of $h=\left(h_{\gamma}\right)_{\gamma \in \Gamma}$ with $\rho_{m}=0$ and $\rho_{j}=\cdots=\rho_{1}=0$.

(3) Assume that $f$ has support in $Z(q)$. If $t_{m-1}, \ldots, t_{j+1}$ are nonzero, the coefficients $b_{l \beta}$ of $\tilde{f}$ in the lower zwickel $Y(r, \ell)$ determine all coefficients of $f$.

This ends the excerpt from [Ha1 about the proof of the Kangaroo Theorem. Actually, the assertions of the theorem are rather straightforward consequences of the above proposition: inverting the transformation matrix between the coefficients vectors of the polynomials allows us to determine the initial form of $g$ as alluded to in assertion (4) of the theorem. As for the proof of the proposition itself, the formula from (1) is an exercise in binomial expansion, assertion (2) is tricky and relies on a special numbering of the lattice points in zwickels in order to make the matrix block diagonal, and (3) follows rather quickly from (2).

I. Oblique polynomials. We now describe the initial form of the polynomials $g$ of the (purely inseparable) equations $x^{p}+y^{r} g(y)=0$ defining wild singularities. In Ha1, the uniqueness assertion (4) of the Kangaroo Theorem was established for the (weighted) initial form of the equation of an arbitrary wild hypersurface singularity, and oblique polynomials were characterized in various specific situations. In [Hi1], a general description of oblique polynomials is given, and Schicho found 
independently a similar formula. Below we combine all viewpoints to a unified presentation.

Fix variables $y=\left(y_{m}, \ldots, y_{1}\right)$. Set $\ell=m-1$, and let $p$ be the characteristic of the ground field $K$. A nonzero polynomial $P=y^{r} g(y)$ with $r \in \mathbb{N}^{m}$ and $g$ homogeneous of degree $k$ is called oblique with parameters $p, r$ and $k$ if $P$ has no nontrivial $p$ th power polynomial factor and if there is a vector $t=\left(0, t_{\ell}, \ldots, t_{1}\right) \in\left(K^{*}\right)^{m}$ so that the polynomial $P^{+}(y)=\left(y+t y_{m}\right)^{r} g\left(y+t y_{m}\right)$ has, after deleting all $p$ th power monomials from it, order $k+1$ with respect to the variables $y_{\ell}, \ldots, y_{1}$. Without loss of generality, the vector $t$ can and will be taken equal to $(0,1, \ldots, 1)$. We shall write $\operatorname{ord}_{z}^{p} P^{+}$to denote the order of $P^{+}$with respect to $z=\left(y_{\ell}, \ldots, y_{1}\right)$ modulo $p$ th powers.

Example 4. Take $m=2, p=2$ and $P(y)=y_{2} y_{1}\left(y_{2}^{2}+y_{1}^{2}\right)$ with $k=2$. Then $P^{+}(y)=P\left(y_{2}, y_{1}+y_{2}\right)=y_{2} y_{1}^{2}\left(y_{1}+y_{2}\right)$ has modulo squares order 3 with respect to $y_{1}$.

It is checked by computation that the condition $\operatorname{ord}_{z}^{p} P^{+} \geq k+1$ on $P^{+}$is a prerequisite for the occurence of a kangaroo point as in the theorem. Moh's result implies $\operatorname{ord}_{z}^{p} P^{+} \leq k+1$, so that equality must hold. Condition (4) of the theorem tells us that there is, up to addition of $p$ th powers, at most one oblique polynomial for each choice of the parameters $p, r$ and $k$. In order that $P$ is indeed oblique it is then also necessary that the degree of $P$ is a multiple of $p$ and that $r$ satisfies $\bar{r}_{m}^{p}+\cdots+\bar{r}_{1}^{p} \leq\left(\phi_{p}(r)-1\right) \cdot p$ (again by the theorem).

We dehomogenize $P$ with respect to $y_{m}$. This clearly preserves $p$ th powers. Moreover, when applied to monomials of total degree divisible by $p$ (as is the case for the monomials of the expansion of $P$ ), the dehomogenization creates no new $p$ th powers. It is thus an "authentic" transformation in our context; i.e., the characterization of oblique polynomials can be transcribed entirely to the dehomogenized situation. Setting $y_{m}=1$ and $z=\left(y_{\ell}, \ldots, y_{1}\right)$, we get $Q(z)=P(1, z)=z^{s} \cdot h(z)$ with $s=\left(r_{\ell}, \ldots, r_{1}\right) \in \mathbb{N}^{\ell}$ and $h(z)=g(1, z)$ a polynomial of degree $\leq k$. The translated polynomial is $Q^{+}(z)=Q(z+\mathbb{I})=(z+\mathbb{I})^{s} \cdot h(z+\mathbb{I})$, where $\mathbb{I}=(1, \ldots, 1) \in \mathbb{N}^{\ell}$. The condition $\operatorname{ord}_{z}^{p} P^{+} \geq k+1$ now reads $\operatorname{ord}_{z}^{p} Q^{+} \geq k+1$ or, equivalently, $Q^{+} \in\left\langle z_{\ell}, \ldots, z_{1}\right\rangle^{k+1}+K\left[z^{p}\right]$. Let us write this as

$$
(z+\mathbb{I})^{s} \cdot h(z+\mathbb{I})-v(z)^{p} \in\left\langle z_{\ell}, \ldots, z_{1}\right\rangle^{k+1}
$$

for some polynomial $v \in K[z]$. As $h$ has degree less than or equal to $k$, the polynomial $v$ cannot be zero. In addition, we see that the condition $\operatorname{ord}_{z}^{p} Q^{+} \geq k+1$ is stable under multiplication with homogeneous $p$ th power polynomials $w(z)$, in the sense that $\operatorname{ord}_{z}^{p}\left(w^{p} \cdot Q^{+}\right) \geq k+1+p \cdot \operatorname{deg} w$. Using that $(z+\mathbb{I})^{s}$ is invertible in the completion $K[[z]]$, we get

$$
h(z+\mathbb{I})=\left\lfloor(z+\mathbb{I})^{-s} \cdot v(z)^{p}\right\rfloor_{k},
$$

where $\lfloor u(z)\rfloor_{k}$ denotes the $k$-jet (that is, expansion up to degree $k$ ) of a formal power series $u(z)$. From Moh's inequality we know that $(z+\mathbb{I})^{s} \cdot h(z+\mathbb{I})-v(z)^{p}$ cannot belong to $\left\langle z_{\ell}, \ldots, z_{1}\right\rangle^{k+2}$. Therefore, in the case that $v(z)$ is a constant, the homogeneous form of degree $k+1$ in $(z+\mathbb{I})^{-s}$ must be nonzero. This form equals $\sum_{\alpha \in \mathbb{N}^{\ell},|\alpha|=k+1}\left(\begin{array}{c}-s \\ \alpha\end{array}\right) z^{\alpha}$. We conclude that if all $\left(\begin{array}{c}-s \\ \alpha\end{array}\right)$ with $|\alpha|=k+1$ are zero in $K$, then $v$ was not a constant 17 Inverting the translation $\tau(z)=z+\mathbb{I}$, we get the

\footnotetext{
${ }^{17}$ The converse need not hold; see the example.
} 
following formula for the dehomogenized initial form at antelope points preceding kangaroo points,

$$
z^{s} \cdot h(z)=z^{s} \cdot \tau^{-1}\left\{\left\lfloor(z+\mathbb{I})^{-s} \cdot v(z)^{p}\right\rfloor_{k}\right\} .
$$

The homogenization of this polynomial with respect to $y_{m}$ followed by the multiplication with $y_{m}^{r_{m}}$ then yields the actual oblique polynomial $P(y)=y^{r} g(y)$.

Example 5. In the example $P(y)=y_{2}^{3} y_{1}^{3}\left(y_{2}^{2}+y_{1}^{2}\right)$ from the beginning, we have characteristic $p=2$, exponents $r_{2}=r_{1}=3$ and degree $k=2$. Therefore, $\ell=1$ and $s=3$, which yields a binomial coefficient $\left(\begin{array}{c}-3 \\ \alpha\end{array}\right)=\left(\begin{array}{c}-3 \\ 3\end{array}\right)=-10$ equal to 0 in $K$. Indeed, $P$ has as a nonmonomial factor $g(y)$ the square $\left(y_{2}+y_{1}\right)^{2}$. In the example $P(y)=y_{2} y_{1}\left(y_{2}^{2}+y_{1}^{2}\right)$ from above with $r_{2}=r_{1}=s=1$, the polynomial $g$ is again a square, even though $\left(\begin{array}{c}-s \\ \alpha\end{array}\right)=\left(\begin{array}{c}-1 \\ 3\end{array}\right)=-1$ is nonzero in $K$.

J. Resolution of surfaces. In the surface case, there are several ways to overcome (or avoid) the obstruction produced by the appearance of kangaroo points. The first proof of surface resolution in positive characteristic is due to Abhyankar, using commutative algebra and field theory [Ab1]. Resolution invariants for surfaces then appear, at least implicitly, in his later work on resolution of three-folds. In Hi4, Hironaka proposes an explicit invariant for the embedded resolution of surfaces in three-space (see $\mathrm{Ha} 4$ for its concise definition). It is not clear how to extend this invariant to higher dimensions.

In [Ha1, it is shown for surfaces that during the blowups prior to the jump at a kangaroo point the shade must have decreased at least by 2 (with one minor exception) and thus makes up for the later increase at the kangaroo point. To be more precise, given a sequence of point blowups in a three-dimensional ambient space for which the subsequent centers are equiconstant points for some $f$, call the antelope point the point $a$ immediately prior to a kangaroo point $a^{\prime}$, and the oasis point the last point $a^{\circ}$ below $a$ where none of the exceptional components through $a$ has appeared yet. The following is then a nice exercise:

Fact. The shade of $f$ drops between the oasis point $a^{\circ}$ and the antelope point $a$ of a kangaroo point $a^{\prime}$ at least to the integer part of its half,

$$
\operatorname{shade}_{a} f \leq\left\lfloor\frac{1}{2} \cdot \operatorname{shade}_{a^{\circ}} f^{\circ}\right\rfloor \text {. }
$$

In the purely inseparable case of an equation of order equal to the characteristic, this decrease thus dominates the later increase of the shade by 1 except for the case shade $_{a^{\circ}} f^{\circ}=2$, which is easy to handle separately and will be left to the reader. It seems challenging to establish a similar statement for singular three-folds in fourspace.

In [HW], we proceeded somewhat differently by also considering blowups after the occurence of a kangaroo point. A detailed analysis shows that when taking three blowups together (the one between the antelope and the kangaroo point, and two more afterward), the shade always either decreases in total or, if it remains constant, an auxiliary secondary shade drops. This shade can again be interpreted as the order of a suitable coefficient ideal (now in just one variable), made coordinate independent by maximizing it over all choices of hypersurfaces inside the chosen hypersurface of weak maximal contact.

The compelling thing is that one can subtract, following an idea of Dominik Zeillinger [Ze] which was made precise and worked out by Wagner, a correction 
term from the shade which eliminates the increases without creating new increases at other blowups. This correction term, called the bonus, is defined in a subtle way according to the internal structure of the defining equation. It is mostly zero and takes, in certain well-defined situations, a value between 0 and $1+\delta$.

This bonus allows us to define an invariant - a triple consisting of the order, the modified shade and the secondary shade - which now drops lexicographically after each blowup. The bonus is defined with respect to a local flag as defined in Ha5]. Flags break symmetries and are stable under blowup (in a precise sense) and thus allow us to define the bonus at any stage of the resolution process. We refer to $\mathrm{HW}$ for the details, as well as for the definition of an alternative invariant, the height, which is even simpler to use for the required induction. It profits much more from the flag than the shade and allows a simpler definition of the bonus. The invariant built from the height yields a quite systematic induction argument which may serve as a testing ground for the embedded resolution of singular three-folds.

K. Example. This is the simplest example for the occurrence of a wild singularity and a kangaroo point in a resolution process. Consider the following sequence of three point blowups in characteristic 2 :

$$
\begin{array}{rlrl}
f^{0} & \left.=x^{2}+1 \cdot\left(y^{7}+y z^{4}\right) \text { (oasis point } a^{0}\right), & (x, y, z) \rightarrow(x y, y, z y), \\
f^{1} & =x^{2}+y^{3} \cdot\left(y^{2}+z^{4}\right), & (x, y, z) \rightarrow(x z, y z, z), \\
f^{2} & \left.=x^{2}+y^{3} z^{3} \cdot\left(y^{2}+z^{2}\right) \text { (antelope point } a^{2}\right), & (x, y, z) \rightarrow(x z, y z+z, z), \\
f^{3} & =x^{2}+z^{6} \cdot(y+1)^{3}\left((y+1)^{2}+1\right) & \\
& \left.=x^{2}+z^{6} \cdot\left(y^{5}+y^{4}+y^{3}+y^{2}\right) \text { (kangaroo point } a^{3}\right) .
\end{array}
$$

The oblique polynomial appears at the antelope point $a^{2}$ in the form $y^{3} z^{3} \cdot\left(y^{2}+z^{2}\right)$. The kangaroo point is a uniquely specified point $a^{3}$ of the exceptional divisor of the third blowup. It is the unique equiconstant point of the exceptional divisor where the shade of $f$ increases. It lies off the transforms of the exceptional components produced by the first two blowups (see Figure 1). The coordinate change $x \rightarrow$ $x+y z^{3}$ at $a^{3}$ eliminates $y^{2} z^{6}$, realizes the shade, and produces

$$
f^{3}=x^{2}+z^{6} \cdot\left(y^{5}+y^{4}+y^{3}\right) .
$$

The order of $f$ has remained constant equal to 2 throughout. But the shade of $f$ has increased between $a^{2}$ and $a^{3}$. Namely, in $y^{3} z^{3} \cdot\left(y^{2}+z^{2}\right)$ the monomial $y^{3} z^{3}$ is exceptional, and the remaining factor $y^{2}+z^{2}$ has order 2 , whereas in $z^{6} \cdot\left(y^{5}+y^{4}+y^{3}\right)$ the exceptional factor is $z^{6}$ and the remaining factor $y^{5}+y^{4}+y^{3}$ has order 3 . Thus shade $_{a^{3}} f^{3}=3>2=\operatorname{shade}_{a^{2}} f^{2}$. Observe that the shade drops between the oasis and antelope point by 3 .

L. Bibliographic comments. We briefly relate the contents of this note to the existing literature on resolution in positive characteristic. The arithmetic condition $\bar{r}_{m}^{p}+\cdots+\bar{r}_{1}^{p} \leq\left(\phi_{p}(r)-1\right) \cdot p$ on the exceptional multiplicities at an antelope point appears in a different perspective also in the work of Abhyankar on good points [Ab2]. Cossart and Moh studied in detail many phenomena related to the appearance of kangaroo points $\mathrm{Co}, \mathrm{Mo}$.

We already mentioned the paper [CJS on the embedded resolution of surfaces of arbitrary codimension. As for dimension three, there are recent results and proofs of Cutkosky, and Cossart and Piltant for the nonembedded resolution of three-folds 
in positive characteristic $\mathrm{Cu}, \mathrm{CP} 1, \mathrm{CP} 2$. Cutkosky reduces Abhyankar's proof (of over 500 pages) of resolution in characteristic greater than 5 to some forty pages, Cossart and Piltant establish the result with considerably more effort for arbitary fields. Both proofs use substantially the embedded resolution of surfaces (built on the invariant from [Hi4]), but they do not provide embedded resolution of three-folds.

As for dimension $n$, Hironaka develops in $\mathrm{Hi1}, \mathrm{Hi2}$, $\mathrm{Hi3}$ an elaborate machinery of differential operators in arbitrary characteristic in order to construct generalizations of hypersurfaces of maximal contact by allowing primitive elements as defining equations. The main difficulty is thus reduced to the purely inseparable case and metastatic points, which precisely correspond to our kangaroo points. Hironaka then asserts that this type of singularities can be resolved directly. There is no written proof of this available at the moment.

There is a novel approach to resolution by Villamayor and his collaborators Benito, Bravo and Encinas [Vi2, Vi3, BV, EV3]. It is based on projections instead of restrictions for the descent in dimension. A substitute for coefficient ideals is constructed via Rees algebras and differential operators, which are called elimination algebras. It provides a new resolution invariant for characteristic $p$ (which coincides with the classical one in zero characteristic). All the necessary properties are proven. This allows us to reduce by blowups to a so-called monomial case (which, however, seems to be still unsolved, and could be much more intricate than the classical monomial case).

In a somewhat different vein, Kawanoue and Matsuki have announced a program for resolution in arbitrary characteristic and dimension [Ka, $\mathrm{MK}$. Again, they use differential operators to define a suitable resolution invariant and then show its upper semicontinuity. The termination of the resulting algorithm seems not to be ensured yet.

Włodarczyk has informed the author that he has recently studied the structure of kangaroo points and that he sees possibilities to define an invariant which does not increase. Again, one has to wait until written material becomes available.

M. Retrospective. It is tempting to try the resolution invariant from characteristic zero also in positive characteristic. After having observed that it may increase in special circumstances, it is also natural to study the cases where this actually happens. This attempt has been presented in this paper. The hope then is that the understanding of the obstruction may allow us to overcome the increase either by extra arguments or by modifying the invariant thus yielding finally a complete resolution. This would be the conservative approach to arbitrary characteristic 18

\footnotetext{
${ }^{18}$ The results of the author's investigations on kangaroo points were written up around 2003 and assembled in the manuscript [Ha1], mostly for personal reference. It was only circulated among the experts working in the field, because the classification of kangaroo points did not apparently show the way toward resolution in positive characteristic (even though a new proof for the surface case was found, as well as several other resolution strategies could be designed). In short, the results were never published.

In fall 2008, Heisuke Hironaka gave several talks at Harvard, the Clay Mathematics Institute and the Research Institute for Mathematical Sciences at Kyoto. There he presented a program for attacking the characteristic $p$ case. In the course of the argument, Hironaka relied on the author's classification of kangaroo points. He then claimed that this classification does indeed pave the way toward resolution in positive characteristic; that it does provide the missing link. At this moment, no complete written proof for the claim is available.
} 
But genuine progress would consist in inventing a new invariant (which never increases). This would be, in the simplest case, a new measure which describes the "distance" of a polynomial to be a monomial (up to coordinate changes and multiplication by units in the formal power series ring). The classical recipe, "factor from the polynomial the exceptional monomial and take the order of the remaining factor as invariant", seems to be just too crude in arbitrary characteristic.

Many important results in mathematics had a hard time becoming generally accepted and understood. The original formulation may have been excessively complicated, with a proof more like a struggle than a concise argument. For the problem of resolution, we have to admit that we are still in the stage of struggling. The available proofs are certainly not the final picture. In such cases it helps to proceed virtually some fifty years (assuming that till then the resolution of singularities has become a well-understood fact) and to look back at our present time. What do we see there?

Inductions! And again inductions!

The main feature of many of the present proofs is indeed a weaving of several intertwined inductions. Induction on the dimension, the local embedding dimension of a singularity, the local multiplicity, a local resolution invariant. So resolution is today a multistep procedure, mostly completely ineffective. And, in fact, already for relatively modest surfaces the resolved variety is covered by hundreds of affine charts (corresponding to dozens of blowups).

The main challenge for us is to better understand algebraic varieties at their singularities. The singular locus is described by certain minors of the Jacobian matrix of the defining equations, but this description is not really convenient here: taking the singular locus with this ideal structure, the induced blowup does in general not yield a resolution. This approach is known as the Nash-modification of the variety. Hironaka and Spivakovsky have shown that surfaces can be resolved by combining them with normalizations Hi6, Sp, GS. There are attempts to refine Nash-modifications by considering higher order jet schemes [Ya], but without definite breakthrough yet.

But notice: from Hironaka's theorem it follows (at least in characteristic zero) that there does exist another ideal structure on the singular locus of a variety so that the induced blowup with this center resolves the singularities in one single stroke. Formidable! The mere existence of this ideal structure is opposed to the (hitherto) failure of describing its structure in concrete examples. It is there, but we don't see it.

Probably we will still have to wait a while for deep understanding of the resolution of singularities - even though a proof for positive characteristic may appear in the near future. We shall keep in touch!

\section{ACKNOWLEDGMENTS}

The author is indebted to many people for sharing their ideas and insights with him. Among them are Heisuke Hironaka, Shreeram Abhyankar, Orlando Villamayor, Santiago Encinas, Ana Bravo, Gerd Müller, Josef Schicho, Gábor Bodnár, Dale Cutkosky, Edward Bierstone, Pierre Milman, Jaroslav Włodarczyk, Bernard Teissier, Vincent Cossart, Mark Spivakovsky, Hiraku Kawanoue, Kenji Matsuki, Li Li, Daniel Panazzolo, Anne Frühbis-Krüger, and János Kollár. It should be clear that many of the concepts and ideas presented in this article have been developed 
over the years by various people working in the field. Precise credits are not always easy to give, and we apologize if some omission or inacurracy has occurred. We thank Dominique Wagner and Eleonore Faber for a careful reading of the text and several substantial improvements, and Rocío Blanco for helpful programming support.

\section{About THE AUthor}

Herwig Hauser studied in Paris with Teissier and Douady. His interests include resolution of singularities, local analytic varieties, infinite dimensional geometry, and visualization of surfaces. He is currently a professor at the University of Vienna.

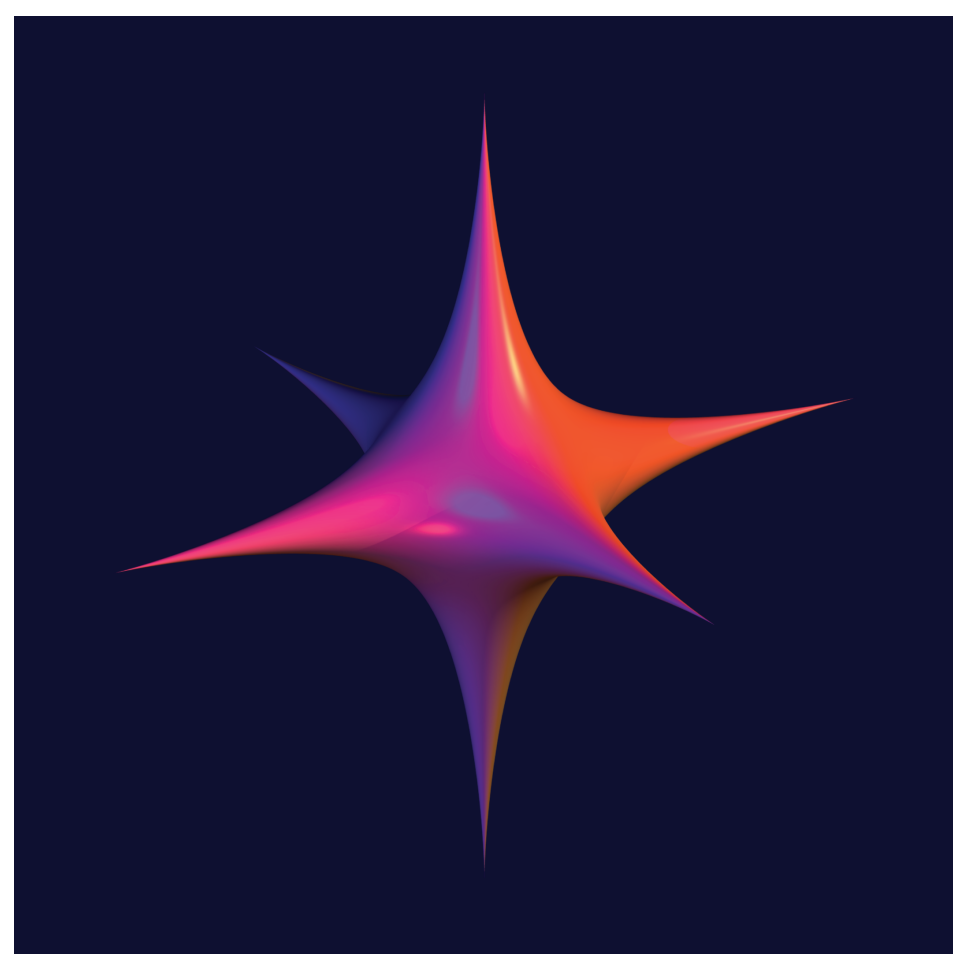

FiguRE 2. The surface "Stern" of equation (1).

\section{REFERENCES}

[Ab1] Abhyankar, S.: Local uniformization of algebraic surfaces over ground fields of characteristic p. Ann. Math. 63 (1956), 491-526. MR0078017 (17:1134d)

[Ab2] Abhyankar, S.: Good points of a hypersurface. Adv. Math. 68 (1988), 87-256. MR.934366 (89e:14012)

[AO] Abramovich, D., Oort, F.: Alterations and resolution of singularities. In: Resolution of Singularities (Obergurgl, 1997, ed. H. Hauser et al.) Progress in Math. 181, Birkhäuser 2000, pp. 39-108. MR.1748617 (2001h:14009)

[Be] Berthelot, P.: Altérations de variétés algébriques (d'après A.J. de Jong). Sém. Bourbaki 1995-96, exp. 815. Astérisque 241 (1997), 273-311. MR.1472543 (98m:14021)

[BM] Bierstone, E., Milman, P.: Canonical desingularization in characteristic zero by blowing up the maximum strata of a local invariant. Invent. Math. 128 (1997), 207-302. MR 1440306 (98e:14010) 
[BV] Bravo, A., Villamayor, O.: Singularities in positive characteristic, stratification and simplification of the singular locus. arXiv:0807.4308

[Co] Cossart, V.: Sur le polyèdre caractéristique. Thèse d'État. 424 pages. Univ. Paris-Sud, Orsay 1987.

[CJS] Cossart, V., Jannsen, U., Saito, S.: Canonical embedded and non-embedded resolution of singularities of excellent surfaces. arXiv:0905.2191

[CP1] Cossart, V., Piltant, O.: Resolution of singularities of three-folds in positive characteristic I. J. Algebra 320 (2008) 1051-1082. MR2427629 (2009f:14024)

[CP2] Cossart, V., Piltant, O.: Resolution of singularities of three-folds in positive characteristic II. J. Algebra (2009), to appear. MR2494751

[Cu] Cutkosky, Resolution of singularities for 3-folds in positive characteristic. Amer. J. Math. 131 (2009), 59-127. MR2488485

[dJ] de Jong, J.: Smoothness, semi-stability and alterations. Publ. Math. IHES 83 (1996), 51-93. MR.1423020(98e:14011)

[EiH] Eisenbud, D., Harris, J.: The geometry of schemes. Graduate Texts in Mathematics. Springer 2000. MR1730819 (2001d:14002)

[EH] Encinas, S., Hauser, H.: Strong resolution of singularities in characteristic zero. Comment. Math. Helv. 77 (2002), 421-445. MR1949115 (2004c:14021)

[EV1] Encinas, S., Villamayor, O.: A course on constructive desingularization and equivariance. In: Resolution of singularities (Obergurgl, 1997, ed. H. Hauser et al.) Progress in Math. 181, Birkhäuser 2000. MR:1748620 (2001g:14018)

[EV2] Encinas, S., Villamayor, O.: Good points and constructive resolution of singularities. Acta Math. 181 (1998), 109-158. MR 1654779 (99i:14020)

[EV3] Encinas, S., Villamayor, O.: Rees algebras and resolution of singularities. Rev. Mat. Iberoamericana. Proceedings XVI-Coloquio Latinoamericano de Álgebra 2006.

[FH] Faber, E., Hauser, H.: Today's menu: Geometry and resolution of singular algebraic surfaces. Bull. Amer. Math. Soc., to appear.

[GS] Gonzalez-Sprinberg, G.: Désingularisation des surfaces par des modifications de Nash normalisées. Sém. Bourbaki 1985/86. Astérisque 145-146 (1987), 187-207. MR880033 (89a:14015)

[Ha1] Hauser, H.: Why the characteristic zero proof of resolution fails in positive characteristic. Manuscript 2003, available at www.hh.hauser.cc.

[Ha2] Hauser, H.: Wild singularities and kangaroo points in the resolution of algebraic varieties over fields of positive characteristic. Preprint 2009.

[Ha3] Hauser, H.: The Hironaka theorem on resolution of singularities. (Or: A proof that we always wanted to understand.) Bull. Amer. Math. Soc. 40 (2003), 323-403. MR1978567 (2004d:14009)

[Ha4] Hauser, H.: Excellent surfaces over a field and their taut resolution. In: Resolution of Singularities (Obergurgl, 1997, ed. H. Hauser et al.) Progress in Math. 181, Birkhäuser 2000. MR 1748627 (2001f:14028)

[Ha5] Hauser, H.: Three power series techniques. Proc. London Math. Soc. 88 (2004), 1-24. MR2063657(2005f:14032)

[Ha6] Hauser, H.: Seven short stories on blowups and resolution. In: Proceedings of Gökova Geometry-Topology Conference 2005, (ed. S. Akbulut et al.) International Press, 2006, pp. 1-48. MR 2282008 (2008a:14023)

[Ha7] Hauser, H.: Seventeen obstacles for resolution of singularities. In: The Brieskorn Anniversary volume, Progress in Math. 162, Birkhäuser 1997. MR1652479 (99g:14016)

[HR] Hauser, H., Regensburger, G.: Explizite Auflösung von ebenen Kurvensingularitäten in beliebiger Charakteristik. L'Enseign. Math. 50 (2004), 305-353. MR2116719|(2006b:14022)

[HW] Hauser, H., Wagner, D.: Two new invariants for the embedded resolution of surfaces in positive characteristic. Preprint 2009.

[Hi1] Hironaka, H.: Program for resolution of singularities in characteristics $p>0$. Notes from lectures at the Clay Mathematics Institute, September 2008.

[Hi2] Hironaka, H.: A program for resolution of singularities, in all characteristics $p>0$ and in all dimensions. Lecture Notes ICTP Trieste, June 2006.

[Hi3] Hironaka, H.: Theory of infinitely near singular points. J. Korean Math. Soc. 40 (2003), 901-920. MR 1996845 (2005a:14003) 
[Hi4] Hironaka, H.: Desingularization of excellent surfaces. Notes by B. Bennett at the Conference on Algebraic Geometry, Bowdoin 1967. Reprinted in: Cossart, V., Giraud, J., Orbanz, U.: Resolution of surface singularities. Lecture Notes in Math. 1101, Springer 1984. MR775681 (87e:14032)

[Hi5] Hironaka, H.: Resolution of singularities of an algebraic variety over a field of characteristic zero. Ann. Math. 79 (1964), 109-326. MR0199184 (33:7333)

[Hi6] Hironaka, H.: On Nash blowing-up. In: Arithmetic and Geometry II. Birkhäuser, 1983, pp. 103-111. MR717608 (84k:14012)

[Ka] Kawanoue, H.: Toward resolution of singularities over a field of positive characteristic. I. Publ. Res. Inst. Math. Sci. 43 (2007), 819-909. MR2361797(2008m:14028)

[Ko] Kollár, J.: Lectures on resolution of singularities. Annals Math. Studies, vol. 166. Princeton 2007. MR 2289519 (2008f:14026)

[Lp1] Lipman, J.: Desingularization of 2-dimensional schemes. Ann. Math. 107 (1978), 151-207. MR0491722 (58:10924)

[Lp2] Lipman, J.: Introduction to resolution of singularities. Proc. Symp. Pure Appl. Math. 29, Amer. Math. Soc. 1975, 187-230. MR0389901 (52:10730)

[MK] Matsuki, K., Kawanoue, H.: Toward resolution of singularities over a field of positive characteristic (The Idealistic Filtration Program) Part II. Basic invariants associated to the idealistic filtration and their properties. arXiv:math/0612008

[Mo] Moh, T.-T.: On a stability theorem for local uniformization in characteristic $p$. Publ. Res. Inst. Math. Sci. 23 (1987), 965-973. MR935710 (89d:14018)

[Mu] Mulay, S.: Equimultiplicity and hyperplanarity. Proc. Amer. Math. Soc. 87 (1983), 407413. MR.715854 (85d:14005)

[Na1] Narasimhan, R.: Monomial equimultiple curves in positive characteristic. Proc. Amer. Math. Soc. 89 (1983), 402-413. MR 715853 (85d:14006)

[Na2] Narasimhan, R.: Hyperplanarity of the equimultiple locus. Proc. Amer. Math. Soc. 87 (1983), 403-406. MR684627 (84f:14001)

[Pa] Panazzolo, D.: Resolution of singularities of real analytic vector fields in dimension three. Acta Math. 197 (2006) 167-289. MR2296055 (2007m:37044)

[Se] Seidenberg, A.: Reduction of singularities of the differential equation $A d y=B d x$. Amer. J. Math. 90 (1968), 248-269. MR0220710 (36:3762)

[Sp] Spivakovsky, M.: Sandwiched singularities and desingularization of surfaces by normalized Nash transformations. Ann. Math. 131 (1990), 411-491. MR.1053487 (91e:14013)

[Vi1] Villamayor, O.: Constructiveness of Hironaka's resolution. Ann. Sci. École Norm. Sup. 22 (1989), 1-32. MR985852 (90b:14014)

[Vi2] Villamayor, O.: Hypersurface singularities in positive characteristic. Adv. Math. 213 (2007), 687-733. MR 2332606 (2008g:14021)

[Vi3] Villamayor, O.: Elimination with applications to singularities in positive characteristic. Publ. Res. Inst. Math. Sci. 44 (2008), 661-697. MR2426361 (2009h:14028)

[Wl] Włodarczyk, J.: Simple Hironaka resolution in characteristic zero. J. Amer. Math. Soc. 18 (2005), 779-822. MR2163383 (2006f:14014)

[Ya] Yasuda, T.: Higher Nash blowups. Compos. Math. 143 (2007), 1493-1510. MR2371378 (2008j:14029)

[Za] Zariski, O.: Reduction of the singularities of algebraic three dimensional varieties. Ann. Math. 45 (1944), 472-542. MR0011006 (6:102f)

[Ze] Zeillinger, D.: Polyederspiele und Auflösen von Singularitäten. PhD Thesis, Universität Innsbruck, 2005.

Fakultät für Mathematik, Universität Wien, Nordbergstrasse 15, A-1090, Wien, AusTRIA

E-mail address: herwig.hauser@univie.ac.at 